SUPPORTING INFORMATION

\title{
The Photophysical and Morphological Implications of Single- Strand Conjugated Polymer Folding in Solution
}

Thomas J. Fauvell, ${ }^{\dagger, \mathrm{a}, \mathrm{b}}$ Tianyue Zheng, ${ }^{\dagger, \mathrm{c}}$ Nicholas E. Jackson, ${ }^{\mathrm{a}}$ Mark A. Ratner, ${ }^{\mathrm{a}}$ Luping $\mathrm{Yu}^{*, \mathrm{c}}$ and Lin X. Chen ${ }^{*, \mathrm{a}, \mathrm{b}}$

${ }^{a}$ Department of Chemistry and the Argonne-Northwestern Solar Energy Research (ANSER) Center, Northwestern University, 2145 Sheridan Road, Evanston, Illinois 60208, United States

${ }^{\mathrm{b}}$ Chemical Sciences and Engineering Division, Argonne National Laboratory, 970o South Cass Avenue, Argonne, Illinois 60439, United States

${ }^{c}$ Department of Chemistry and Jams Frank Institute, The University of Chicago, 929 East $57^{\text {th }}$ Street, Chicago, Illinois 60637, United States

\section{$\underline{\text { Table of Contents }}$}

1. Thermodynamics of Conjugated Polymer Folding (1-6)

2. Molecular Dynamics Simulations (7-10)

3. Quantum-Chemical Calculations (11-13)

4. Low MW Polymer Fluorescence Spectra (14-15)

5. Synthesis of PTB7 Oligomers (15-18)

6. HNMR Data (19-24)

\section{The Thermodynamics of Conjugated Polymer Folding}

Our goal is to compute the critical length, denoted by $\mathrm{N}_{\text {crit }}$ repeat units, of an arbitrary conjugated polymer at which folding becomes thermodynamically favorable. "Folding" is a term that has many definitions, so here we choose a very simple definition of folding: the change of state from a single extended ideal chain of $\mathrm{N}$ repeat units with energetics dominated by polymer-solvent interactions, to a state where the polymer is folded perfectly in half into a highly non-covalently "cross-linked" ideal loop possessing both enthalpic self(polymer-polymer)-interactions and polymer-solvent interactions. For considering entropic contributions to this process, it is useful to think of the process as a three-step cycle occurring in accordance with Figure S1 below: the initial ideal chain is converted into an ideal loop, which is finally converted into a cross-linked ideal loop. 


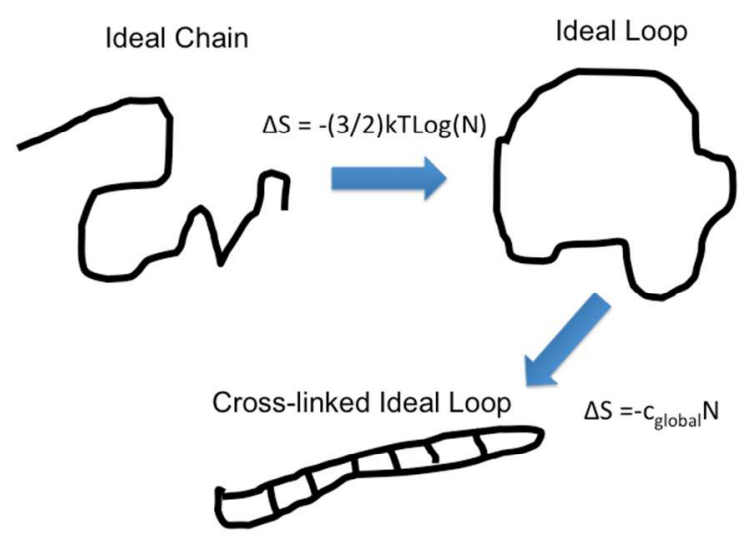

Fig S1 - Schematic for the folding process. "Cross-linked" here does not mean chemical crosslinking, and in our specific case denotes a non-covalent force, i.e. $\pi-\pi$ stacking.

Our analysis begins by examining the free-energy change, $\Delta F=F_{f}-F_{u}=\Delta H_{f}-T \Delta S_{f}$, associated with the folding process. The enthalpy change of this process is denoted $\Delta \mathrm{H}_{\mathrm{f}}$ (Eq. 1), for which we use a mean-field style interaction parameter with linear chain length dependence $\left(\Delta w ; \Delta w=w_{f}-w_{u}\right)$ to take into account intermolecular interactions, as well as an enthalpy penalty associated with the intramolecular cost of breaking conjugation and forming the kink necessary for folding, $H_{k i n k}$. (Note: negative $\Delta w$ denotes a situation where enthalpic polymerpolymer interactions are stronger than enthalpic polymer-solvent interactions, and $H_{k i n k}$ is always positive.)

$$
\Delta H_{f}=N \Delta w+H_{k i n k}
$$

There should be two main entropic penalties associated with folding: the restriction of global motion (ideal chain -> cross-linked ideal loop) and the restriction of intramolecular degrees of freedom. We define the latter entropic penalty in the internal degrees of freedom as $T \Delta S_{\text {int }}$, which we assume is linearly dependent on the number of repeat units $\mathrm{N},\left(\Delta s_{\text {int }}=\frac{\Delta S_{\text {int }}}{N}\right)$ taking into account 4-body dihedral motion, 3-body angle bends, and 2-body bond stretches. Practically speaking, we expect the contributions from the dihedral degrees of freedom to be the largest, as the effective dihedral potential will dramatically increase upon chain folding, whereas the other degrees of freedom should not experience significant restrictions of their phase space (Eq. 2).

$$
T \Delta S_{\text {int }}=N T \Delta s_{\text {int }} \approx N T \Delta s_{\text {dih }}
$$

Next we estimate the global chain conformational entropy penalty associated with the folding of a polymer from an extended chain into a highly cross-linked ideal chain. We note that this is a difficult problem to which current solutions are actively being sought. Higher-level theoretical approaches have been used to compute the entropy reduction associated with an ideal cross- 
linking polymer chain converting from a closed ideal loop with no cross links to a closed loop with $\mathrm{N} / 4$ cross-links. ${ }^{1}$ The results of these higher-level approaches yield an entropy reduction that is approximately linear in the number of cross-links. Assuming that in the case of our folded polymer the number of cross-links $=$ (number of repeat units) $/ 2$, then we can conservatively estimate the entropy penalty associated with this restriction to be in the range of $0.1-0.5 \mathrm{kcal} / \mathrm{mol}$ per repeat unit based on reference 1 . This, when combined with the total entropy penalty associated with converting an ideal chain of length $\mathrm{N}$ to a perfect loop of length $\mathrm{N}$, originally derived by Flory $\left(\Delta \mathrm{S}=-(3 / 2) \mathrm{kT} \log (\mathrm{N})^{2}\right)$, is suggestive that the entropic penalty associated with folding is at most $\sim 1 \mathrm{kcal} / \mathrm{mol}$ per repeat unit. Since this derivation is heavily qualitative, we utilize these facts and make the simple assumption that the global free-energy change associated with the entropy reduction of chain folding is linearly proportional to the number of repeat units (Eq. 3).

$$
T \Delta S_{\text {chain }}=N T \Delta s_{\text {global }}
$$

Now, taking into account all of the relevant terms in the free-energy, we can write down a freeenergy expression and, solving for the value of $\mathrm{N}$ at which the free-energy change is zero, we arrive at Eq. 4.

$$
N_{c r i t}\left(\Delta w-T \Delta s_{\text {int }}\right)+H_{\text {kink }}=T \Delta s_{\text {global }} N_{\text {crit }}
$$

Which is rearranged to form Eq. 5.

$$
N_{\text {crit }}=\frac{H_{\text {kink }}}{T \Delta s_{\text {global }}+T \Delta s_{\text {int }}-\Delta w}
$$

Note: An ideal chain is a reasonable approximation for the behavior of a conjugated polymer as long as a monomer length is chosen that approximates the number of monomers involved in the Kuhn length of that material.

\section{Interpretation of this result (Eq. 5):}

- There are four terms contributing to the critical folding length of the polymer: 1) The global free-energy penalty associated with folding $T \Delta s_{\text {global }}$ (ideal $->$ loop -> crosslinked loop) 2) The enthalpic penalty of forming the kink required for folding $\left.\left(H_{k i n k}\right) 3\right)$ The entropic penalty of freezing out internal degrees of freedom upon folding $\left(T \Delta s_{\text {int }}\right)$ and 4) The change in enthalpy that results from replacing a portion of polymer-solvent interactions with polymer-polymer interactions $(\Delta w)$.

- If $\mathrm{N} \leq 0$, then folding of the polymer into an aggregated state is not possible.

- If folding is possible, then the following expected behaviors are reproduced: 
1. As the enthalpic penalty associated with forming the kink increases (e.g. the polymer has stronger and stronger conjugation), then the critical length at which the polymer will fold increases.

2. If the entropic penalty associated with the freezing out of the internal degrees of freedom via folding becomes too negative, then $\mathrm{N}_{\text {crit }}$ becomes negative, and folding is not possible.

3. Folding is only possible when $\Delta w$ is negative, suggesting that folding is only possible when the enthalpic polymer-polymer self-interaction is more favorable than the polymer-solvent interaction.

\section{What is the magnitude of $T \Delta s_{i n t}$ ?}

We operate under the assumption that the primary contribution to the entropic penalty of the internal degrees of freedom is associated with the freezing out of the dihedral degrees of freedom. To model this process we make three assumptions about the nature of the dihedral degrees of freedom, namely:

1. The dihedrals can be treated as uncoupled, non-interacting degrees of freedom $\left(Z_{\text {poly-dih }}=Z_{\text {dih }}^{N-1}\right) ; T \Delta S_{\text {int }}$ should be linear in $\mathrm{N}$.

2. The dihedral potential of a conjugated polymer can be qualitatively modeled with a simple cosine term, where $V_{b}$ is the dihedral barrier height and $\phi$ is the dihedral angle (Eq. 6).

$$
V(\phi)=V_{b}(1-\operatorname{Cos}[\phi])
$$

3. The free-energy contribution resulting from the folding restriction can be modeled as the change in free-energy resulting from changing the dihedral potential from $V_{u} \rightarrow V_{f}$, where $V_{f}>V_{u}$.

We begin by writing down the partition function associated with a single dihedral degree of freedom in the canonical ensemble (Eq. 7).

$$
Z_{\text {dih }}=\int_{0}^{2 \pi} e^{-\frac{V_{b}(1-\operatorname{Cos}[\phi])}{k_{B} T}} d \phi
$$

Which possesses an analytical solution from which we can write down $\mathrm{Z}_{\text {poly-dih }}$ as Eq. 8 .

$$
Z_{\text {poly-dih }}=\left[2 \pi e^{-\frac{V_{b}}{k_{B} T}} I_{0}\left(\frac{V_{b}}{k_{B} T}\right)\right]^{N-1}
$$


where $\mathrm{I}_{0}$ is a modified Bessel function of the first kind. Now, a simple free energy difference can be taken between a polymer in the extended state and a polymer in the folded state (Eq. 9):

$$
\Delta F_{\text {dih }}=-k_{B} T \ln \left(\left[2 \pi e^{-\frac{V_{\text {fold }}}{k_{B} T}} I_{0}\left(\frac{V_{\text {fold }}}{k_{B} T}\right)\right]^{N-1}\right)+k_{B} T \ln \left(\left[2 \pi e^{-\frac{V_{\text {extend }}}{k_{B} T}} I_{0}\left(\frac{V_{\text {extend }}}{k_{B} T}\right)\right]^{N-1}\right)
$$

or more simply as Eq. 10.

$$
\Delta F_{\text {dih }}=k_{B} T(N-1)\left[\ln \left(2 \pi e^{-\frac{V_{\text {extend }}}{k_{B} T}} I_{0}\left(\frac{V_{\text {extend }}}{k_{B} T}\right)\right)-\ln \left(2 \pi e^{-\frac{V_{\text {fold }}}{k_{B} T}} I_{0}\left(\frac{V_{\text {fold }}}{k_{B} T}\right)\right)\right]
$$

Evaluating this expression directly provides a rough approximation for the free-energy loss associated with dihedral freezing, which is plotted below.

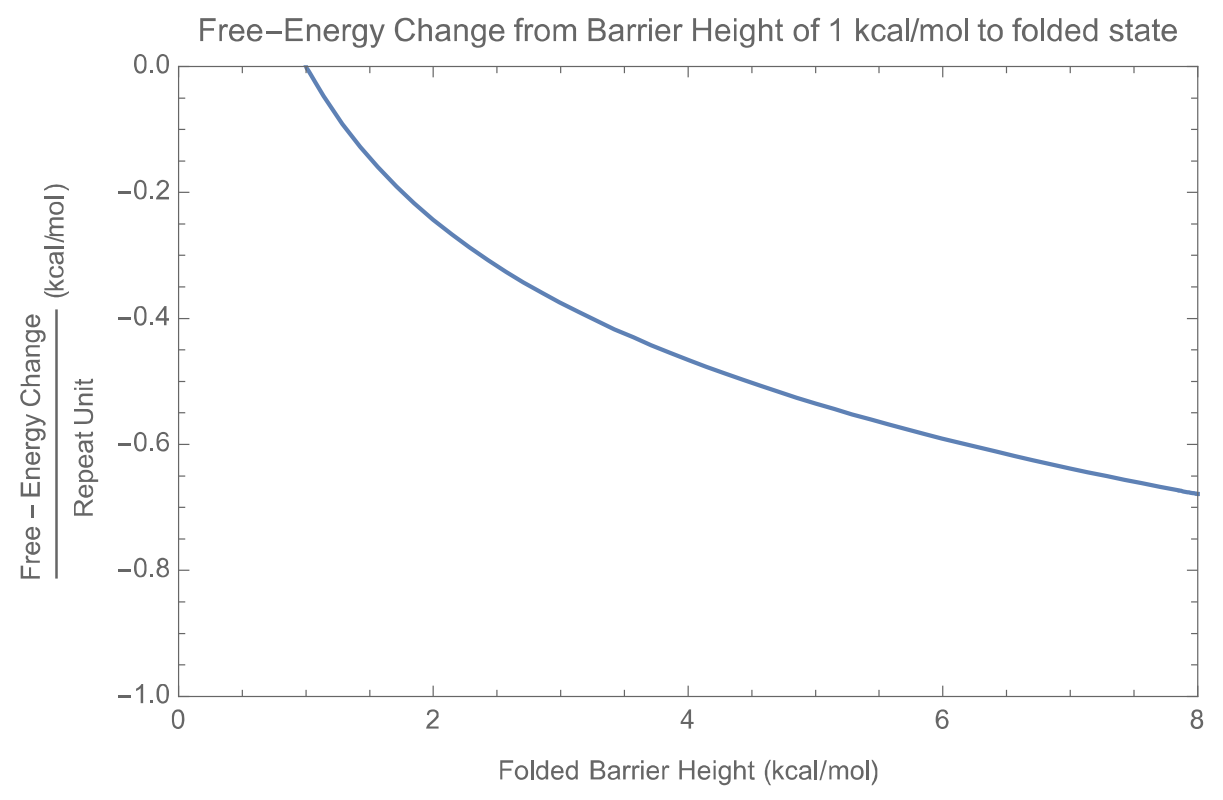

Fig S2 - Free-energy penalty associated with freezing the dihedral degrees of freedom.

This tells us that the entropic contribution from freezing out the dihedral degrees of freedom is rather small, especially since most unfolded polymer chains have dihedral potentials significantly larger than $1 \mathrm{kcal} / \mathrm{mol}$. There is some element of underestimation in this approach as the minimum energy states are not necessarily accessible and could be kinetically trapped and not thermodynamically accessible. Regardless, this value is likely significantly less than the enthalpy gain of conjugated polymer folding for PTB7. .

\section{What is the magnitude of $H_{k i n k}$ ?}


Molecular dynamics simulations identify the enthalpic penalty of kink formation as $\sim 28 \mathrm{kcal} / \mathrm{mol}$ for PTB7. $\mathrm{H}_{\text {kink }}$ should display a dependence on the molecular structure of the polymer repeat unit, likely increasing with increasing $\pi$-system size.

\section{$\underline{\text { A Microscopic Model for Conjugated Polymers }}$}

To deduce relationships between molecular structure and polymer folding thermodynamics, we require a microscopic connection between the enthalpy of folding and the molecular structure. Experimentally, it is commonly observed that the more $\pi$-electrons a repeat unit of a polymer possesses, the less soluble that polymer becomes due to solution aggregation. Consequently, we define an empirical term to describe the free-energy change of the polymer backbone upon folding as a function of the number of $\pi$-electrons in a repeat unit (Eq. 11).

$$
\Delta w=A_{\pi}\left(\sigma_{\pi-\pi}-\sigma_{\pi-s o l v}\right)
$$

where $\sigma$ is a solvation enthalpy per surface area of the polymer repeat unit and $A_{\pi}$ is the accessible surface area of the conjugated polymer repeat unit $\pi$-system. We write $A_{\pi}$ as Eq. 12 .

$$
A_{\pi}=n_{\pi} \alpha_{\pi}
$$

where $\alpha_{\pi}$ is the effective surface area $\pi$-electron. With this in mind we can write the expression for $\mathrm{N}_{\text {crit }}$ (Eq. 13).

$$
N_{c r i t}=\frac{H_{k i n k}}{T \Delta s_{i n t}-n_{\pi} \alpha_{\pi}\left(\sigma_{\pi-\pi}-\sigma_{\pi-s o l v}\right)+T \Delta s_{g l o b a l}}
$$

This result suggests that for solution aggregates where side-chain degrees of freedom and enthalpic interactions remain constant in the folded and unfolded states, the critical length of folding for a polymer should depend approximately inversely on the number of $\pi$-electrons in the repeat unit of the polymer chain. The difference in the solvation enthalpy, $\sigma$, values represents the microscopic difference between the polymer-polymer and polymer-solvent enthalpic interactions. If the solvent interactions are more favorable than the polymer-polymer, then the denominator of the above expression is negative, and the polymer cannot fold. As the $\pi$-system becomes larger, the difference in solvation energy between the polymer-polymer and polymersolvent interactions becomes larger, and the critical length of folding decreases.

Moreover, for $\pi$-bonding backbones, it is apparent that the entropic terms in the free-energy are significantly less than the enthalpic terms, which is suggestive that one can simply write the critical fold length as Eq. 14. 


$$
N_{c r i t}=\frac{H_{k i n k}\left(n_{\pi}\right)}{n_{\pi} \alpha_{\pi}\left(\sigma_{\pi-\pi}-\sigma_{\pi-s o l v}\right)}
$$

Where $\boldsymbol{H}_{\boldsymbol{k i n k}}\left(\boldsymbol{n}_{\boldsymbol{\pi}}\right)$ denotes a functional dependence of the kink penalty enthalpy on the size of the $\pi$-electron system of the backbone repeat unit.

\section{Molecular Dynamics Simulations}

\section{Representative Trajectories for Unfolded and Folded Conformations}

We perform 50 trajectories for each oligomer length in an attempt to observe folding. Figure S3 represents example trajectories exhibiting energy conservation. The fraction of trajectories that fold as a function of oligomer length is the following:

4 mer $-0 / 50$

$6 \mathrm{mer}-0 / 50$

$8 \mathrm{mer}-17 / 50$

$10 \mathrm{mer}-23 / 50$

12 mer $-36 / 50$

It is apparent that by 8 repeat units folding is enthalpically favorable, and the reason that we do not observe $100 \%$ of trajectories folding for lengths of 8,10 , and 12 is that the trajectories are kinetically trapped, and $25 \mathrm{~ns}$ is not enough time to observe formation of this state.

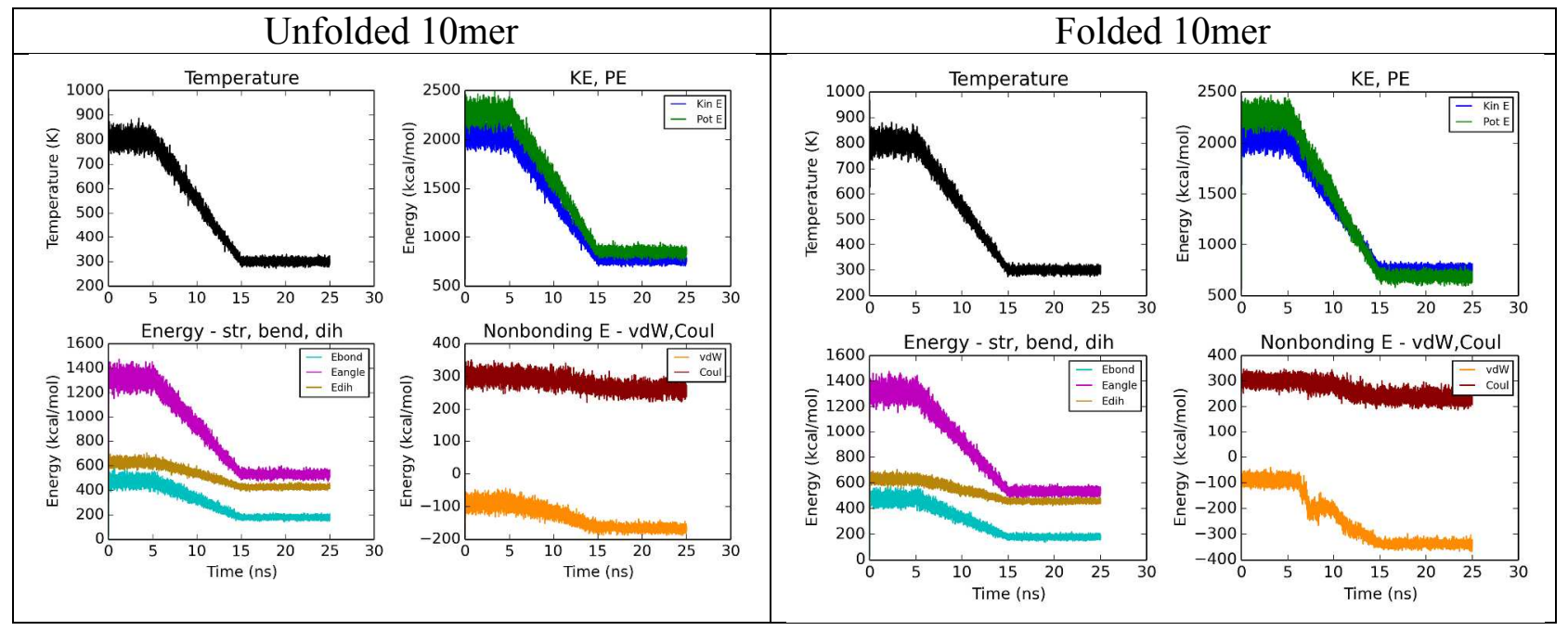

Fig S3 - Representative trajectories of unfolded and folded 10mers. Folded trajectories are easily visible as having a significantly larger magnitude of the van der Waals interactions relative to the unfolded trajectories.

\section{Calculation of $\Delta H_{\text {fold }}$ per repeat unit}


We compute $\Delta \mathrm{H}_{\text {fold }}$ averaged over all folding trajectories for oligomers of length 8,10 and 12 . We then plot these three data points (Fig S4) and since it is approximately linear, we deduce a $\Delta \mathrm{H}_{\text {fold }}$ per repeat unit. We then decompose $\Delta \mathrm{H}_{\text {fold }}$ into all of its contributions.

$\Delta \mathrm{H}_{\text {fold }}=-16.9+/-8.1 \mathrm{kcal} / \mathrm{mol}$

An alternative identification of $\Delta \mathrm{H}_{\text {fold }}$ can be computed by using only the $8 \mathrm{mer}$, since it will have the most consistent fold style, compared to the longer strands, where many different types of folds can be defined. If examination of only the 8 mer is performed, similar values for $\Delta \mathrm{H}_{\text {fold }}$ are determined but with smaller error. We stick with the larger error value since this is a vacuum calculation and solvent effects are left out, making the exact value unreliable. To get an experimentally relevant value of $\Delta \mathrm{H}_{\text {fold }}$ explicit solvent calculations MUST be performed.

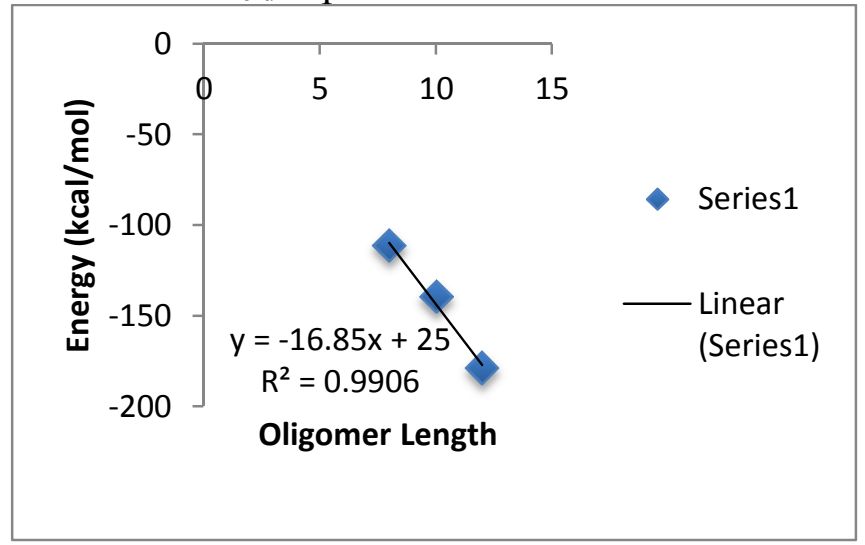

Fig S4 - Fitting to determine $\Delta \mathrm{H}_{\text {fold }}$

\section{Calculation of $\Delta H_{\text {kink }}$}

We decompose $\Delta \mathrm{H}_{\text {fold }}$ into contributions arising from all of its intramolecular components(2body bond stretches, 3-body angle bends, 4-body dihedral twists, and improper bends):

$\Delta \mathrm{H}_{\mathrm{kink}}=28.1+/-5.2 \mathrm{kcal} / \mathrm{mol}$

$\Delta \mathrm{H}_{\text {kink-bond }}=-0.1+/-0.2 \mathrm{kcal} / \mathrm{mol}$

$\Delta \mathrm{H}_{\text {kink-ang }}=3.5+/-1.0 \mathrm{kcal} / \mathrm{mol}$

$\Delta \mathrm{H}_{\text {kink-dih }}=23.4+/-2.3 \mathrm{kcal} / \mathrm{mol}$

$\Delta \mathrm{H}_{\text {kink-imp }}=1.3+/-0.2 \mathrm{kcal} / \mathrm{mol}$

From these values it is apparent that the 4-body dihedral penalties correspond to the largest contribution to the value of $\Delta \mathrm{H}_{\text {kink }}$, followed by the 3-body angle bends. We expect this value of $\mathrm{H}_{\text {kink }}$ to be relevant both in vacuum and in solvent, making this calculation useful for understanding the intramolecular energy penalty associated with folding.

Calculation of $T \Delta S_{\text {dih }}$ and $T \Delta S_{\text {global }}$

Computation of these quantities is outlined in the previous section on the thermodynamics of conjugated polymer folding

Enthalpic Averages over all trajectories (all energies in $\mathrm{kcal} / \mathrm{mol}$ ):

4mer - $0 / 50$ folded

unfolded/folded averages 
vdw avg $\mathrm{E}=-64.4+/-1.5$

coul avg $\mathrm{E}=133.6+/-3.0$

$\operatorname{ang} \operatorname{avg} \mathrm{E}=211.8+/-0.5$

bond avg $\mathrm{E}=71.0+/-0.3$

imp avg $E=6.4+/-0.1$

$\operatorname{dih} \operatorname{avg} E=169.6+/-1.0$

6mer $-0 / 50$ folded

unfolded averages

vdw avg $\mathrm{E}=-98.4+/-1.8$

coul avg $\mathrm{E}=154.1+/-5.5$

$\operatorname{ang} \operatorname{avg} E=317.8+/-0.6$

bond avg $\mathrm{E}=106.9+/-0.3$

imp avg $E=9.6+/-0.1$

dih avg $E=254.8+/-1.1$

8mer - 4/50 folded

unfolded

vdw avg $\mathrm{E}=-132.1+/-2.0$

coul avg $\mathrm{E}=158.7+/-4.5$

ang avg $\mathrm{E}=423.4+/-0.6$

bond avg $\mathrm{E}=142.5+/-0.3$

$\operatorname{imp} \operatorname{avg} E=12.8+/-0.1$

$\operatorname{dih} \operatorname{avg} E=340.1+/-1.5$

\section{folded}

vdw avg $\mathrm{E}=-260.9+/-5.9$

coul avg $\mathrm{E}=149.8+/-4.1$

$\operatorname{ang} \operatorname{avg} \mathrm{E}=426.3+/-0.8$

bond avg $\mathrm{E}=142.4+/-0.1$ 
imp avg $E=14.4+/-0.4$

dih avg $E=362.9+/-1.9$

10mer - 16/50 folded

unfolded

vdw avg $E=-165.9+/-2.4$

coul avg $\mathrm{E}=262.7+/-5.1$

$\operatorname{ang} \operatorname{avg} \mathrm{E}=529.4+/-0.8$

bond avg $\mathrm{E}=178.4+/-0.4$

imp avg $E=16.0+/-0.1$

dih avg $E=424.7+/-1.4$

\section{folded}

vdw avg $\mathrm{E}=-326.6+/-9.7$

coul avg $\mathrm{E}=253.7+/-11.0$

ang $\operatorname{avg} \mathrm{E}=532.3+/-1.4$

bond avg $\mathrm{E}=178.1+/-0.4$

$\operatorname{imp} \operatorname{avg} E=17.4+/-0.2$

$\operatorname{dih} \operatorname{avg} E=450.7+/-6.8$

12mer - 21/50 folded

unfolded averages

vdw avg $E=-199.9+/-2.4$

coul avg $\mathrm{E}=282.3+/-6.5$

ang $\operatorname{avg} \mathrm{E}=635.0+/-1.2$

bond avg $\mathrm{E}=213.9+/-0.5$

imp avg $E=19.2+/-0.1$

dih avg $E=510.0+/-1.1$

\section{folded}

vdw avg $\mathrm{E}=-398.3+/-22.1$ 
coul avg $\mathrm{E}=274.1+/-9.5$

ang avg $\mathrm{E}=639.6+/-1.9$

bond avg $\mathrm{E}=214.0+/-0.6$

imp avg $\mathrm{E}=20.5+/-0.4$

dih avg $\mathrm{E}=531.5+/-9.4$

\section{Quantum-Chemical Calculations}

To assess the effect of aggregation-induced planarization on the optical gap shift of PTB7, we perform quantum-chemical calculations on two representative geometries of a PTB7 tetramer. One geometry represents the structure prior to aggregation-induced planarization, with all dihedral angles at their minima (non-planar $\sim 30$ degrees). The other geometry represents the structure following aggregation-induced planarization, with all dihedral angles constrained to be planar (planar). Both structure were geometry optimized at the B3LYP/6-31G** level of theory, with the planar structure possessing constraints holding all intermonomer dihedral angles planar during optimization, whereas the non-planar structure was free from geometry constraints. All geometry optimizations were performed using QCHEM 4.0. The absolute energies of the energy-minimized geomerties are presented in Table S1. The geometries for both structures are provided below Table S1.

The shift in the lowest absorption energy between the planar and non-planar structures was computed in ORCA 3.0.3 using three levels of theory: B3LYP/def2-SVP, CAM-B3LYP/def2SVP, and ZINDO/S, the results of which are presented below in Table S1.

\begin{tabular}{|c|c|c|c|c|}
\hline & $\begin{array}{c}\text { Ground-State } \\
\text { Energy } \\
(\text { Hartree })\end{array}$ & $\begin{array}{c}\text { B3LYP } \\
\text { Optical Gap } \\
\left(\mathrm{cm}^{-1}\right)\end{array}$ & $\begin{array}{c}\text { CAM-B3LYP } \\
\text { Optical Gap } \\
\left(\mathrm{cm}^{-1}\right)\end{array}$ & $\begin{array}{c}\text { ZINDO/S } \\
\text { Optical Gap } \\
\left(\mathrm{cm}^{-1}\right)\end{array}$ \\
\hline Non-Planar & -11050.1190123429 & 15531.8 & 20309.1 & 16709.3 \\
\hline Planar & -11050.1133125222 & 14472.6 & 19036.4 & 15634.4 \\
\hline Energy Shift & $\mathbf{0 . 0 0 5 7}$ & $\mathbf{1 0 5 9 . 2}$ & $\mathbf{1 2 7 2 . 7}$ & $\mathbf{1 0 7 4 . 9}$ \\
\hline
\end{tabular}

Table S1 - Calculated ground state and excited state energies of the non-planar and planar geometries of a PTB7 tetramer.

\section{Planar XYZ Geometry}

$\begin{array}{llll}\mathrm{C}-0.0157739682 & -0.8733174330 & 0.1100517109 \\ \mathrm{C}-1.1929300417 & -1.5853228274 & 0.1200408342 \\ \mathrm{C}-2.3602482977 & -0.7628359384 & 0.1303242069 \\ \mathrm{H}-1.2492914090 & -2.6654559128 & 0.0958303706 \\ \mathrm{C}-2.0403603724 & 0.6256323848 & 0.1146280866 \\ \mathrm{C}-3.7122391801 & -1.1600335799 & 0.1475379444 \\ \mathrm{C}-3.0088085999 & 1.6202182291 & 0.1040317622 \\ \mathrm{C}-4.3617496342 & 1.2227986808 & 0.0865283861 \\ \mathrm{C}-4.6809424400 & -0.1654986531 & 0.1156406732\end{array}$

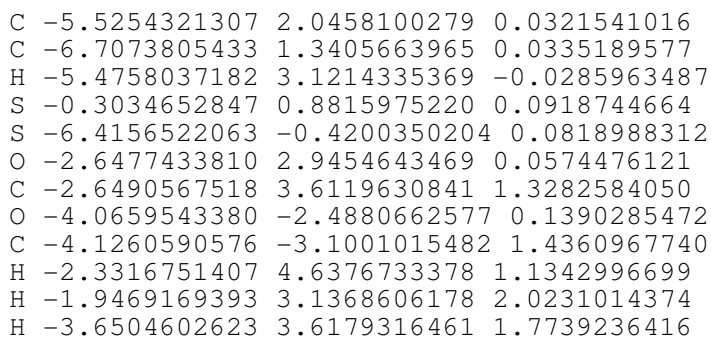

C $-5.5254321307 \quad 2.04581002790 .0321541016$ C $-6.7073805433 \quad 1.3405663965 \quad 0.0335189577$

$\mathrm{H}-5.47580371823 .1214335369-0.0285963487$

$\mathrm{S}-0.3034652847 \quad 0.8815975220 \quad 0.0918744664$

S $-6.4156522063-0.4200350204 \quad 0.0818988312$

$\begin{array}{lllll}\mathrm{O} & -2.6477433810 & 2.9454643469 & 0.0574476121\end{array}$

C $-2.6490567518 \quad 3.6119630841 \quad 1.3282584050$

$\mathrm{O}-4.0659543380-2.4880662577 \quad 0.1390285472$

C $-4.1260590576-3.1001015482 \quad 1.4360967740$

$\mathrm{H}-2.3316751407 \quad 4.6376733378 \quad 1.1342996699$

$\mathrm{H}-1.9469169393 \quad 3.1368606178 \quad 2.0231014374$

H $-3.6504602623 \quad 3.6179316461 \quad 1.7739236416$ 
$\mathrm{H}-4.4168317233-4.1383478428 \quad 1.2696677246$ $\mathrm{H}-4.8723259752-2.6079719463 \quad 2.0703868402$ $\mathrm{H}-3.1507108110-3.0695336250 \quad 1.9355745031$ C $-8.5962303523 \quad 3.1298934597-0.0513053506$ C $-10.02877078693 .1515629716-0.0932317398$ C $-10.63515675131 .9063863912-0.0899099464$ C $-8.09525527974 .4743295695-0.0424569955$ C $-9.05413209795 .4475898867-0.0774220798$ F $-6.7883878324 \quad 4.7588682887 \quad 0.0028801677$

S $-9.38374372650 .6818039871-0.0299008382$ C $-8.06215356341 .8315387428-0.0172409460$ S $-10.6892744754 \quad 4.7766050975-0.1244492493$ C $-8.9404773705 \quad 6.9124057370-0.0804013341$ o $-9.90941459827 .6490965463-0.1061830064$ $0-7.66298451567 .3398763840-0.0522632342$ C $-7.5023648323 \quad 8.7692852753-0.0611370352$ $\mathrm{H}-7.9408864937 \quad 9.1989249568-0.9649298447$ $\mathrm{H}-6.4267237876 \quad 8.9401225919-0.0373260488$ $\mathrm{H}-7.9836047379 \quad 9.2151733262 \quad 0.8125975737$

$\mathrm{H}-14.9315664364 \quad 4.2132339774 \quad 1.5688927904$

H $-14.3009562520-2.1560842017-1.9585006533$ S $-18.43787272121 .9472010133-0.2565599326$ C $-18.9569178078 \quad 0.2407686518-0.2012761177$ C $-15.8711320943 \quad 4.3508665260 \quad 1.0210338818$ C $-16.75031244591 .4721382135-0.1996705744$ C $-17.8795523059-0.6106762168-0.1542970871$ C $-15.6588864800 \quad 2.3315969592-0.1905909370$ C $-16.61577204870 .0548544991-0.1630125365$ o $-15.8296726923 \quad 3.6940687766-0.2548910770$ C $-14.37200174691 .7593570703-0.1534514118$ C $-15.3283101433-0.5172805463-0.1274018070$ C $-13.1065699382 \quad 2.4223857547-0.1555233956$ C $-14.2368479652 \quad 0.3417273418-0.1176368145$ C $-15.1218066930-2.5360428457-1.3396451355$ S $-12.5479504575-0.1371390772-0.0564260308$ C $-12.03304148331 .5645240696-0.1022656179$ o $-15.1535027891-1.8792107027-0.0636788926$ $\mathrm{H}-16.0185863541 \quad 5.4116641023 \quad 0.8128512913$

$\mathrm{H}-17.9715646279-1.6839010521-0.0959257486$ $\mathrm{H}-13.0195631986 \quad 3.4990358036-0.2162995864$ $\mathrm{H}-14.9622675169-3.5954437715-1.1329964032$ $\mathrm{H}-16.7023472508 \quad 3.9777064513 \quad 1.6300487965$

$\mathrm{H}-16.0690277841-2.4067313144 \quad-1.8760687894$ C $-20.3696917070-0.0694475292-0.1950138493$ S $-21.53673249831 .2392976227-0.2419103820$ C $-21.0641274052-1.2866774429-0.1646317800$ C $-20.7512582327-2.6863794161-0.1314260764$ C $-22.9057524818 \quad 0.1846403232-0.2146827415$ C $-22.4942539116-1.1177474017-0.1735316305$ C $-21.8341119858-3.5212664427-0.1105801453$ S $-23.3715234598-2.6426786293-0.1334808824$ $\mathrm{F}-19.4931470949-3.1432520225-0.1245491278$ C $-21.9127709068-4.9881255292-0.0727926180$ o $-20.7011365356-5.5785763290-0.0583949258$ C $-20.7276751524-7.0159906009-0.0165500616$ $0-22.9690462954-5.5932895116-0.0560341577$ $\mathrm{H}-21.2408002964-7.3647610663 \quad 0.8827119727$ $\mathrm{H}-19.6829949684-7.3248599765-0.0072566916$ $\mathrm{H}-21.2407063739-7.4166273194-0.8940674105$ o $21.5522465601 \quad 1.5973474992-0.2843480000$ S $23.9542045344-0.4277874345-0.3205794462$ C $21.2275294588 \quad 0.2621619637-0.2519171960$ $\mathrm{H} \quad 21.8981319031 \quad 3.2670978506 \quad 0.8185532395$

C $22.2198101969-0.7130206533-0.2801463945$ C $24.2539100569-2.1553241789-0.3113424510$ H $3.0359189595-9.0036712078-0.5461647235$ H $18.7332011674 \quad 1.7173408653-0.2457129460$ H $21.0825747892-4.4227898612-2.0740239423$ C $21.6205369873 \quad 2.2280925264 \quad 1.0028614594$ C $18.7041295789 \quad 0.6396977548-0.2143053513$ C $19.8857072135-0.1628254263-0.2291585767$ H $19.3469327159-4.0010808315-2.1105142064$ C $21.9252645255-2.1071598690-0.2789849199$ C $23.1221904050-2.9056798968-0.2898116938$ H $8.1975151282-3.8939170145 \quad 1.8957106400$ C $20.1379685725-4.4775788917-1.5201495712$ C $3.5584150248-8.59815802390 .3233388299$ H $4.6038993972-8.9041343497 \quad 0.3209580230$ $\mathrm{H} \quad 16.1108101793 \quad 7.7681927992-0.9600403248$ C $17.5377255710-0.0872936118-0.1794920689$ C $19.5936468728-1.5566535679-0.2271032122$

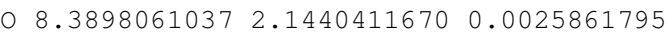
C $16.1726980924 \quad 0.3799209230-0.1578848182$ S $14.8716973492-0.7925027468-0.1271252098$ C $20.5837824555-2.5308839615-0.2575223941$ C $15.6166678434 \quad 1.6689471254-0.1414064720$ H $11.1952994428 \quad 1.9749150488-0.0949196267$ H $9.9631332766-3.62468362591 .9790529020$ O $3.5807007854-7.1604130196 \quad 0.2740522793$ C $11.1229000550 \quad 0.8970020838-0.0396214140$ C $16.0947138903 \quad 3.0220401215-0.1324580814$ $\mathrm{H} \quad 22.3791095746 \quad 1.7530131211 \quad 1.6353593700$ C $14.1835564322 \quad 1.6661852789-0.1105188855$ F 17.3967191029 3.3299768551 -0.1493476758 C $13.59855768210 .4109506970-0.1004244083$ C $8.57474871150 .7827950051 \quad 0.0456995778$ C $9.1492151926-4.0340559314 \quad 1.3697379719$ H $20.6514120090 \quad 2.1941257073 \quad 1.5140217395$ C $15.11914347213 .9783956173-0.0995166215$ S $13.49533337373 .2793660979-0.0756373259$ C $12.2064818558 \quad 0.0500094233-0.0498813991$ C $2.3681008240-6.57352416520 .2663008725$ H $23.1150367201-3.9883094047-0.2680199962$ S $5.8008219977 \quad 0.3776178859 \quad 0.0568041151$ $\begin{array}{llll}\text { C } 9.8665183461 & 0.2214397452 & 0.0174160363\end{array}$ C $15.2066500278 \quad 5.4448466255-0.0787283286$ F $4.7793674634-4.70961465330 .1851344341$ $\begin{array}{llll}0 & 16.4758505274 & 5.8961729998 & -0.1064804413\end{array}$ S $17.8635327201-1.8433618428-0.1743148980$ C $16.6095690890 \quad 7.3283449875-0.0932428551$ O $1.3126067655-7.1792286480 \quad 0.3019275506$ C $7.4916580482-0.0858653397 \quad 0.0648354444$ o $14.2243838065 \quad 6.1631470798-0.0402347864$ C $2.4430549747-5.10727013680 .2103661929$ C $3.5180323664-4.2642615011 \quad 0.1733745172$ $\mathrm{H} \quad 19.8791934525-5.5227256954-1.3424881314$ H $17.6814615117 \quad 7.5196117916-0.1281081462$ C $10.0162503497-1.1955440477 \quad 0.0384293425$ O $20.2587839320-3.8678914076-0.2269944221$ S $11.7107500115-1.6575878561-0.0117792920$ $\mathrm{H} \quad 8.22616730963 .8489989209 \quad 1.0940721452$ C $7.6388626611-1.50287263810 .0798354981$ C $5.2953865085-1.33413614320 .0854787102$ C $3.1912204050-2.8682116825 \quad 0.1256670240$ C $8.9330133524 \quad-2.0636853346 \quad 0.0805012421$ S $0.9060819199-4.2337987546 \quad 0.1873869852$ H $3.0569474881-8.9434914004 \quad 1.2304656666$ $\begin{array}{llll}\text { C } 8.3925110701 & 2.7878644856 & 1.2854049921\end{array}$ H $9.3191293745-5.0989273325 \quad 1.2033522158$ C $3.8883365878-1.64986675410 .0892175379$ C $6.3823468399-2.1788282971 \quad 0.0839833575$ C $1.7672163035-2.70655638350 .1294636583$ S 2.7249655954 $-0.3402229544 \quad 0.0654500248$ $\begin{array}{lllll}0 & 9.1196285736 & -3.4252577659 & 0.0707079966\end{array}$ C $1.3255864511-1.3939627323 \quad 0.0980886130$ H $6.2998832153-3.2541753830 \quad 0.0676376417$ H $16.1724353073 \quad 7.7462381430 \quad 0.8167996203$ H $9.3546631837 \quad 2.6548982917 \quad 1.7938884899$ $\mathrm{H} \quad 7.5895869905 \quad 2.4003302095 \quad 1.9229715013$ $\mathrm{H}-23.9070351385 \quad 0.5891044417-0.2306505194$ H $25.2763197381-2.5087781559-0.3217085275$

\section{Non-Planar XYZ Geometry}

C $-0.0219125880-0.4683344695 \quad 0.2979888139$ C $-1.1397047883-1.17394678200 .6718082716$ C $-2.3531900467-0.4299076570 \quad 0.5495276450$ $\mathrm{H}-1.1100024147-2.1780772495 \quad 1.0751308785$ C $-2.1240602756 \quad 0.9008078580 \quad 0.0937967581$ C $-3.6664745853-0.8473435431 \quad 0.8383998071$ C $-3.14881820371 .8241989131-0.0708884433$ C $-4.46477998681 .4060962759 \quad 0.2146547652$ C $-4.69181534990 .0744544943 \quad 0.6696591926$ C $-5.68047087412 .1444882039 \quad 0.0828555013$ C $-6.7985617811 \quad 1.4343626231 \quad 0.4463714250$ $\mathrm{H}-5.72045167893 .1530702802-0.3018385680$ S $-0.41892666241 .1822451553-0.2281838296$ S $-6.3938691708-0.2141944373 \quad 0.9835613333$ O $-2.8792448056 \quad 3.0876793062-0.5399985042$ C $-2.8151342034 \quad 4.1038933325 \quad 0.4720579654$ 
O $-3.9287039601-2.11176888251 .3093324069$ C $-4.0707576541-3.1132702668 \quad 0.2900705877$ $\mathrm{H}-2.5700835978 \quad 5.0325763970-0.0456647784$ H $-2.0337381879 \quad 3.8768455636 \quad 1.2062121370$ $\mathrm{H}-3.7758177365 \quad 4.2160149009 \quad 0.9872695626$ $\mathrm{H}-4.2799022557-4.0491258483 \quad 0.8102867735$ $\mathrm{H}-3.1502149182-3.2183717252-0.2954000667$ $\mathrm{H}-4.9020826169-2.8742753039-0.3829612087$ C $-8.7477701340 \quad 3.1294377797 \quad 0.4821321721$

C $-10.1780467900 \quad 3.1161601613 \quad 0.4200070352$

C $-10.7416852177 \quad 1.8573447354 \quad 0.3125379976$

C $-8.2749501756 \quad 4.46976901310 .6699566907$

C $-9.2571600854 \quad 5.4177477536 \quad 0.7289302350$

F $-6.9724831556 \quad 4.7542075271 \quad 0.7770445816$

S $-9.4527980721 \quad 0.6679958624 \quad 0.2522940895$

C $-8.1771467368 \quad 1.8551208765 \quad 0.4164839244$

S $-10.8787134729 \quad 4.7191015595 \quad 0.5716622634$

C $-9.1799184227 \quad 6.8751269144 \quad 0.8964488539$

$\begin{array}{llll}0 & -10.1653746789 & 7.5882314176 & 0.9438376251\end{array}$

$\begin{array}{llll}0 & -7.9126745409 & 7.3245716850 & 0.9872945461\end{array}$

C $-7.7852074170 \quad 8.7482118948 \quad 1.1511207079$

H $-8.2092963671 \quad 9.2727211496 \quad 0.2915056149$

H $-6.7148711132 \quad 8.9365979710 \quad 1.2261231730$

$\mathrm{H}-8.2998732506 \quad 9.0780875279 \quad 2.0563718264$

$\mathrm{H}-15.1101686113 \quad 4.3576652060 \quad 1.0426176807$

$\mathrm{H}-14.4123162955-2.6249103068-1.1272829526$

S $-18.4887235847 \quad 1.6746634005-0.6001553839$

C $-18.9980322150 \quad 0.0080369133 \quad-0.2394893880$

C $-16.0225190145 \quad 4.3608471302 \quad 0.4353826521$

C $-16.8147901873 \quad 1.2637210682-0.2661877568$

$\begin{array}{lllll}\text { C } & -17.9344728951 & -0.7929134479 & 0.0892111833\end{array}$

C $-15.7350455078 \quad 2.1371528186-0.3185766910$

C $-16.6762016210-0.1154942930 \quad 0.0642368425$

$\begin{array}{llll}0 & -15.9144154792 & 3.4535722856 & -0.6721052235\end{array}$

C $-14.4551555425 \quad 1.6185752801-0.0429431346$

C $-15.3954503693-0.6345185018 \quad 0.3381949411$

C $-13.1947436245 \quad 2.2934753869 \quad-0.0720516847$

C $-14.3164992905 \quad 0.2384746234 \quad 0.2840930397$

C $-15.2018371060-2.8740057360-0.4090831270$

S $-12.6405415452 \quad-0.1804262730 \quad 0.6020899431$

C $-12.1309458429 \quad 1.4835317738 \quad 0.2425428858$

$\begin{array}{lllll}0 & -15.2058274286 & -1.9497752045 & 0.6897105364\end{array}$

$\mathrm{H}-16.1685130493 \quad 5.3521684648 \quad 0.0037679873$

H $-18.0422003989-1.8305742458 \quad 0.3690450464$

$\mathrm{H}-13.0918993866 \quad 3.3291007431 \quad-0.3707249383$

H $-15.0050366505-3.8566542233 \quad 0.0224837585$

$\mathrm{H}-16.8797168917 \quad 4.1073350404 \quad 1.0697064246$

$\mathrm{H}-16.1692709209-2.8891877171-0.9233625765$

C $-20.3972829131-0.3486316637-0.3058238920$

S $-21.6372447670 \quad 0.8653394210-0.0690040835$

C $-21.0097683864-1.5836278265-0.5249334097$

C $-20.5896669295-2.9235178420-0.8141580697$

C $-22.9383785088-0.2586514939-0.2765461084$

C $-22.4462512702-1.5144241352-0.5012328776$

C $-21.6086368579-3.8196885979-0.9776510783$

S $-23.2086148564-3.0727748633-0.8038918046$

F $-19.2973315666-3.2588140573-0.9078954997$

C $-21.5799100754-5.2604658073-1.2637966094$

O $-20.3272966774-5.7494006040-1.3644330904$

C $-20.2484464396-7.1588401927-1.6401266725$

O $-22.5879564902-5.9307904669-1.3906637547$

$\mathrm{H}-20.7268228974-7.7335219931-0.8434263678$

$\mathrm{H}-19.1839883589-7.3851212854-1.6911873005$

H $-20.7382476584-7.3932763278-2.5881146998$

o $21.53616231660 .8606523556-1.5445986818$

S $23.9058964539-1.0962978675-0.9262073852$

C $21.2180838253-0.2720251183-0.8341136556$

H $21.98153300332 .8390655947-1.4402681553$

C $22.1979311101-1.2108105346-0.5256387090$

$\begin{array}{llll}\text { C } 24.2106801310 & -2.6297023172 & -0.1320069242\end{array}$

H $3.1652614454-8.4750526394-0.8178085234$

H $18.7237612277 \quad 1.1596274010-1.2229590584$

H $20.8415268207-5.2539598351-0.1287079238$

C $21.6954168172 \quad 2.0447631672-0.7489663148$ $\begin{array}{lllll}\text { C } & 18.7216647484 & 0.2407889258 & -0.6554349218\end{array}$ C $19.8966793419-0.5426894736-0.4315511051$ H $19.1232411627-4.7670553951-0.1762417480$ C $21.9124895853-2.41451885850 .1813622088$ C $23.0979445172-3.2020670741 \quad 0.3952432769$ H $9.5886004775-3.9911469570-0.3585684029$ C $19.9780898785-4.96300903630 .4812423441$ C $3.6909809182-8.11472554590 .0694543728$ H $4.7414969500-8.4012818554 \quad 0.0370656964$ H $16.7004255849 \quad 7.4364340945-1.7513804329$ C $17.5839619978-0.3051219402-0.1162441772$ C $19.6137190735-1.7451531911 \quad 0.2784213696$

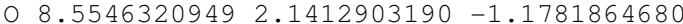
C $16.2368965305 \quad 0.2082546389-0.1616149204$ S $14.8750810797-0.8804268374-0.0143769263$ C $20.5920716619-2.6838807277 \quad 0.5837434017$ C $15.76093293491 .5122095275-0.3271754114$ H $11.38890562551 .8651097485-1.0074821590$ H $7.8474441051-4.0567702488 \quad 0.0407826459$ $\begin{array}{lllll}0 & 3.6875784042 & -6.6765360083 & 0.1097561584\end{array}$ C $11.2374184287 \quad 0.9348262395-0.4746534165$ C $16.33683917552 .8207448238-0.4389460511$ H $22.4831998815 \quad 1.9100688050 \quad 0.0009352895$ C $14.3321292713 \quad 1.6030847464-0.3113135124$ F $17.66001869193 .0155066948-0.4603973179$ C $13.6777306366 \quad 0.3980801064-0.1304907198$ C $8.6789714113 \quad 0.9274713992-0.5459648905$ C $8.8589244166-4.0092842463 \quad 0.4590788931$ H $20.76015321122 .3180173133-0.2471240740$ C $15.4289509154 \quad 3.8382335403-0.5210884416$ S $13.7557330572 \quad 3.2564194322-0.4521016554$ $\begin{array}{lllll}\text { C } 12.2649258407 & 0.1314106229 & -0.0431157787\end{array}$ C $2.4641626410-6.11325909010 .1532812865$ H $23.0985557165-4.14153401770 .9337294193$ S $5.8894357494 \quad 0.6213874125 \quad-0.5882766396$ $\begin{array}{lllll}\text { C } & 9.9399098631 & 0.3860868434 & -0.2265564176\end{array}$ C $15.6199524903 \quad 5.2886729932-0.6545573006$ F $4.8450245711-4.18873899290 .1193189731$

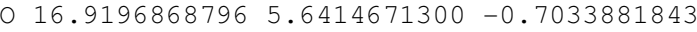
S $17.9147418315-1.8490337818 \quad 0.7086606037$ C $17.1598188888 \quad 7.0535841550-0.8371226321$ $\begin{array}{lllll}0 & 1.4207692407 & -6.7402928203 & 0.1606024181\end{array}$ C $7.5523416044 \quad 0.1704692414-0.2478769493$ ○ $14.69306583496 .0755051466-0.7148844000$ $\begin{array}{lllll}\text { C } 2.5126018336 & -4.6453247791 & 0.1940649295\end{array}$ C $3.5718791343-3.78275954050 .1827409891$ H $19.7291839487-5.77197773351 .1700955572$ H $18.2431919316 \quad 7.1607075791-0.8767920743$ C $10.0119342589-0.8953346460 \quad 0.3933297430$ o $20.2781678028-3.82020530331 .2945526898$ S $11.6737983353-1.36587795390 .7082547936$ H $8.5169144770 \quad 4.1589462015 \quad-0.9464992739$ $\begin{array}{lllll}\text { C } 7.6261198979 & -1.1103973540 & 0.3730442484\end{array}$ C $5.3028005251-0.89090660610 .1448580936$ $\begin{array}{llll}\text { C } 3.2142436461 & -2.3951652826 & 0.2478314646\end{array}$ $\begin{array}{lllll}\text { C } 8.8871276720 & -1.6515761141 & 0.6932296873\end{array}$ $\begin{array}{lllll}\text { S } & 0.9567832079 & -3.8008575888 & 0.2726469171\end{array}$ $\mathrm{H} 3.2066138893-8.52466982820 .9588208311$ C $8.6296104575 \quad 3.2812811790-0.3083361151$ H $9.0343353722-4.8854986732 \quad 1.0851498509$ C $3.8900113453-1.17223819800 .2029758182$ C $6.3343929025-1.6728912999 \quad 0.6020494545$ C $1.7907057642 \quad-2.2556366587 \quad 0.2920215170$ $\begin{array}{llll}\text { S } 2.7203293100 & 0.1292166212 & 0.2416559299\end{array}$

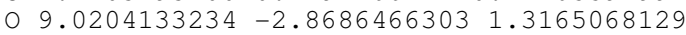
C $1.3353743750-0.9491862424 \quad 0.2790773295$ H $6.1797735227 \quad-2.6126921986 \quad 1.1109574444$ H $16.7502829050 \quad 7.5952547287 \quad 0.0188428566$ H $9.5956000655 \quad 3.3260276256 \quad 0.2070934090$ H $7.8245100261 \quad 3.2625485300 \quad 0.4353000797$ H $-23.9631918464 \quad 0.0778188401-0.2215445332$ $\mathrm{H} 25.2217346667-3.0138013273-0.1070352753$ 


\section{Low MW PTB7 Concentration Dependent Fluorescence}

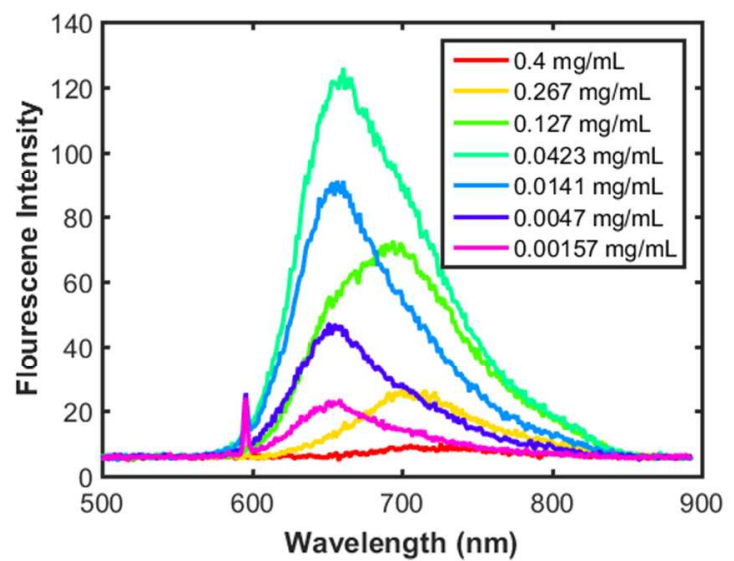

Figure S5. 7 repeating unit polymer

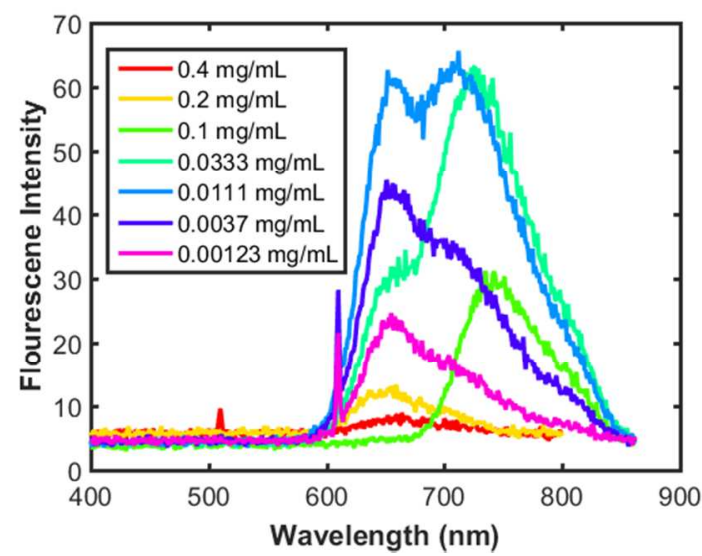

Figure S6. 13 repeating unit polymer

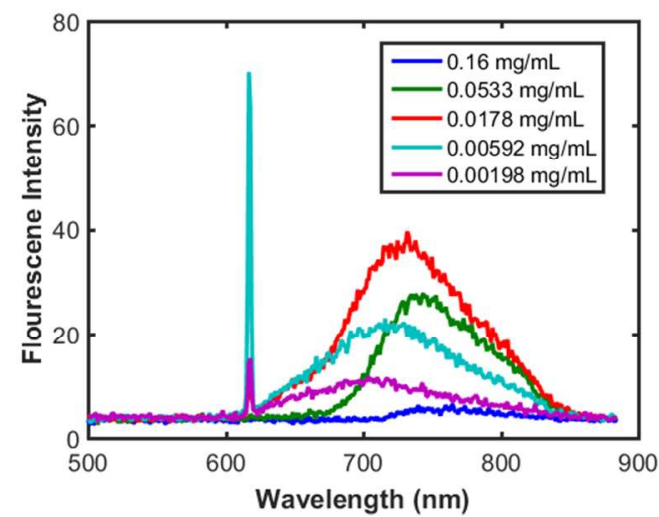

Figure S7. 23 repeating unit polymer 


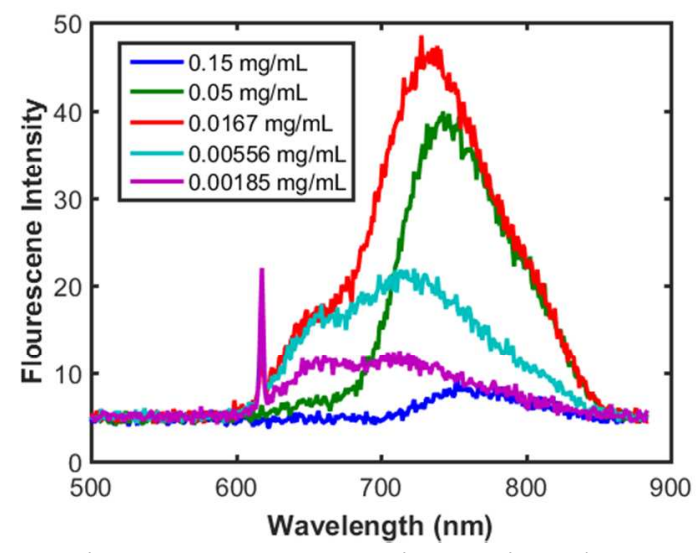

Figure S8. 28 repeating unit polymer

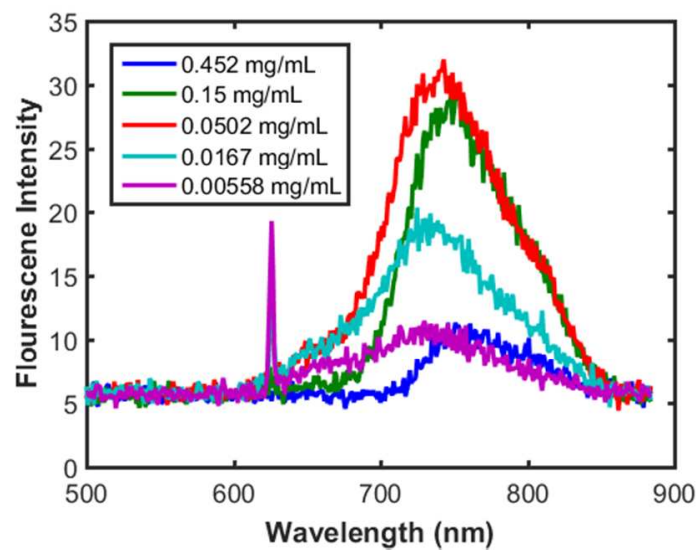

Figure S9. 40 repeating unit polymer

\section{Synthesis of PTB7 oligomers}

Unless otherwise stated, all chemicals obtained from commercial suppliers were used without further purification. All solvents were purified with a standard distillation procedure prior to use. All reactions were carried out under argon atmosphere. 1H, 13C and 19F NMR were recorded by using $500 \mathrm{MHz}$ Bruker DRX-500 spectrometers in $\left(\mathrm{CDCl}_{3}\right)$ with TMS as internal reference, $19 \mathrm{~F}$ NMR also used $\mathrm{C}_{6} \mathrm{~F}_{6}$ (neat, $\delta=-164.9$ ) as external standards; chemical shifts $(\delta$ ) are reported in parts per million. Mass spectra were obtained on a Bruker Daltonics UltrafleXtreme MALDI-TOF system, a Reflective Positive (RP) method using dithranol as matrix. Column chromatography was carried out on silica gel (silica 60M, 400-230 mesh). The 2'-ethylhexyl-4,6-dibromo-3-fluorothieno[3,4-b]thiophene-2-carboxylate and the 2,6bis(trimethyltin)-4,8-bis(2-ethylhexyloxy)benzo [1,2-b:4,5-b']dithiophene were synthesized according to the reported procedures. ${ }^{3}$ M2b were reported in our previous study. ${ }^{3}$ 
<smiles>Oc1c2ccsc2c(O)c2ccsc12</smiles><smiles></smiles><smiles>[R2]Oc1c2ccsc2c([R20])c2cc([Si](C)(C)C)sc12</smiles>

$R=$ 2-ethylhexyl TIPS = triisopropylsilyI<smiles>[R7]Oc1c2ccsc2c(O[R])c2ccsc12</smiles>

$\underset{\text { 2) }}{\stackrel{\text { TIPSCI}}{1 \text { eq BuLi }}}$<smiles>[R]Oc1c2cc(SC(C)C)sc2c([R20])c2ccsc12</smiles><smiles>[R7]Oc1c2cc([Si](C)(C)C)sc2c(OC)c2cc(S[13CH3])sc12</smiles><smiles>[R]Oc1c2cc([R])sc2c(O)c2cc([Si](C)(C)C)sc12</smiles>

1a, $R^{\prime}=H$

$1 \mathrm{~b}, \mathrm{R}^{\prime}=$ TIPS

1c, $\mathrm{R}^{\prime}=\mathrm{SnMe}_{3}$

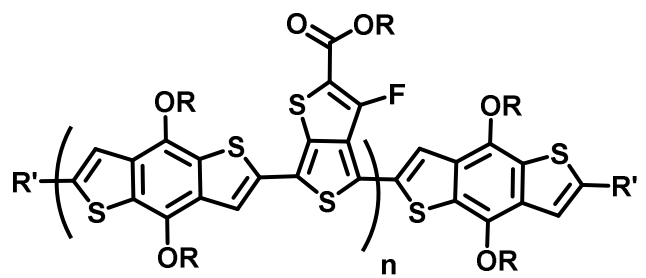<smiles>[R]OC(=O)c1sc2c(Br)c(C(=O)O[R])sc2c1F</smiles>

M1, R'=TIPS

$R=$ 2-ethylhexyl TIPS = triisopropylsilyl

$\mathrm{n}=1$

M2a, $\mathrm{R}^{\prime}=\mathrm{H}$

$M 2 b, R^{\prime}=$ TIPS $M 3, n=2, R^{\prime}=$ TIPS

M2c, $R^{\prime}=\mathrm{SnMe}_{3} \mathrm{M} 4, \mathrm{n}=3, \mathrm{R}^{\prime}=$ TIPS
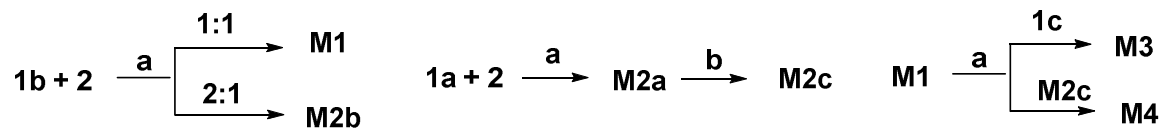

a: $\mathrm{Pd}\left(\mathrm{PPh}_{3}\right)_{4}, \mathrm{LiCl}, \mathrm{Tol} / \mathrm{DMF}, 100^{\circ} \mathrm{C}, \mathrm{b}$ : (1) LDA, THF, $-78^{\circ} \mathrm{C}, 1 \mathrm{~h}$; (2) $\mathrm{Me}_{3} \mathrm{SnCl}, \mathrm{THF},-78^{\circ} \mathrm{C}, 3 \mathrm{~h}$

2-trimethyltin-4,8-bis(2-ethylhexyloxy)benzo[1,2-b:4,5-b']dithiophene

(1a) 4,8-bis(2ethylhexyloxy)benzo [1,2-b:4,5-b']dithiophene $(1.89 \mathrm{~g}, 4.24 \mathrm{mmol})$ was weighed into a $100 \mathrm{~mL}$ round bottom flask and protected with Ar. $50 \mathrm{~mL}$ anhydrous THF was then added and the solution was cooled down to $-78{ }^{\circ} \mathrm{C}$. While stirring, n-BuLi $(2.5 \mathrm{M}, 1.65 \mathrm{~mL})$ was added dropwise. After $30 \mathrm{~min}, \mathrm{Me}_{3} \mathrm{SnCl}(1 \mathrm{M}, 4.6 \mathrm{~mL})$ was added dropwise. The solution was kept in $78{ }^{\circ} \mathrm{C}$ for another $30 \mathrm{~min}$ and then room temperature for 6 hours. The reaction mixture was then poured into water and extracted with $\mathrm{Et}_{2} \mathrm{O}$ and washed with $100 \mathrm{~mL}$ water three times. The organic layer was collected and dried over anhydrous $\mathrm{Na}_{2} \mathrm{SO}_{4}$. After filtration and removal of solvent, the crude product was obtained as light yellow oil $(2.52 \mathrm{~g}, 4.13 \mathrm{mmol})$, and used without further purification. ${ }^{1} \mathrm{HNMR}\left(\mathrm{CDCl}_{3}\right): \delta 0.49-0.55(9 \mathrm{H}, \mathrm{t}), 0.92-0.96(6 \mathrm{H}, \mathrm{m}), 1.00-1.04(6 \mathrm{H}, \mathrm{m})$, 1.36-1.90 $(21 \mathrm{H}, \mathrm{m}), 4.16-4.19(4 \mathrm{H}, \mathrm{m}), 7.33-7.34(1 \mathrm{H}, \mathrm{d}, \mathrm{J}=5.5 \mathrm{~Hz}), 7.45-7.46(1 \mathrm{H}, \mathrm{d}, \mathrm{J}=5.5 \mathrm{~Hz})$, 7.49-7.55 (1H, m).

2-trimethyltin-6-triisopropylsilyl-4,8-bis(2-ethylhexyloxy)benzo[1,2-b:4,5-b']dithiophene (1b) 4,8-bis(2-ethylhexyloxy)benzo [1,2-b:4,5-b']dithiophene (4.24g, 9.49mmol) was weighed into a $250 \mathrm{~mL}$ round bottom flask and protected with Ar. $100 \mathrm{~mL}$ anhydrous THF was then added and the solution was cooled down to $-78{ }^{\circ} \mathrm{C}$. While stirring, $\mathrm{n}-\mathrm{BuLi}(2.5 \mathrm{M}, 3.8 \mathrm{~mL})$ was added dropwise. After $30 \mathrm{~min}$, TIPSCl $(2.25 \mathrm{~g}, 11.3 \mathrm{mmol})$ was added dropwise. The solution was kept in $-78{ }^{\circ} \mathrm{C}$ for another $30 \mathrm{~min}$ and then room temperature for 6 hours. The reaction mixture was then poured into water and extracted with $\mathrm{Et}_{2} \mathrm{O}$. The organic layer was collected and purified 
by column chromatography on silica gel with hexanes as eluent. The 2-triisopropylsilyl-4,8bis(2-ethylhexyloxy)benzo[1,2-b:4,5-b']dithiophene was obtained as pale yellow oil (4.96g, 8.23mmol, 87\%). ${ }^{1} \mathrm{HNMR}\left(\mathrm{CDCl}_{3}\right): \delta 0.88-0.94(6 \mathrm{H}, \mathrm{m}), 1.00-1.04(6 \mathrm{H}, \mathrm{m}), 1.16-1.18(18 \mathrm{H}, \mathrm{d}$, $\mathrm{J}=7.5 \mathrm{~Hz}), 1.36-1.85(21 \mathrm{H}, \mathrm{m}), 4.18-4.21(4 \mathrm{H}, \mathrm{t}, \mathrm{J}=6 \mathrm{~Hz}), 7.34-7.35(1 \mathrm{H}, \mathrm{d}, \mathrm{J}=5.5 \mathrm{~Hz}), 7.46-7.47$ $(1 \mathrm{H}, \mathrm{d}, \mathrm{J}=5.5 \mathrm{~Hz}), 7.63(1 \mathrm{H}, \mathrm{s})$. Treat this oil with similar method as for $\mathbf{1 a}, \mathbf{1 b}$ was obtained as light brown gel $\left(6.28 \mathrm{~g}\right.$, quan), and used without further purification. ${ }^{1} \mathrm{HNMR}\left(\mathrm{CDCl}_{3}\right): \delta 0.49$ $0.55(9 \mathrm{H}, \mathrm{t}), 0.94-0.97(6 \mathrm{H}, \mathrm{m}), 1.00-1.04(6 \mathrm{H}, \mathrm{m}), 1.16-1.18(18 \mathrm{H}, \mathrm{d}, \mathrm{J}=7.5 \mathrm{~Hz}), 1.36-1.90(21 \mathrm{H}$, $\mathrm{m}), 4.19-4.21(4 \mathrm{H}, \mathrm{m}), 7.45-7.54(1 \mathrm{H}, \mathrm{m}), 7.62(1 \mathrm{H}, \mathrm{s})$.

(M1) 1 b $(0.73 \mathrm{~g}, 0.96 \mathrm{mmol})$ and 2'-ethylhexyl-4,6-dibromo-3-fluorothieno[3,4- $b$ ] thiophene-2carboxylate (BrFTTBr) $(0.42 \mathrm{~g}, 0.90 \mathrm{mmol})$ were measured into a round bottom flask, together with $\mathrm{Pd}\left(\mathrm{PPh}_{3}\right)_{4}(45.6 \mathrm{mg})$ and $\mathrm{LiCl}(1 \mathrm{mg})$. After three cycles of vacuum and refill with Ar, toluene/DMF $(40 \mathrm{~mL} / 10 \mathrm{~mL})$ was added. The solution was then heated to $100{ }^{\circ} \mathrm{C}$ o.n. After cooling down to room temperature, the solvent was removed and the reaction mixture was purified by column chromatography of silica gel with hexane/dichloromethane $=2 / 1$ to give the final product M1 as orange oil $\left(0.52 \mathrm{~g}, 0.52 \mathrm{~mol}\right.$, yield 58\%). ${ }^{1} \mathrm{HNMR}\left(\mathrm{CDCl}_{3}\right): \delta 0.90-1.02(12 \mathrm{H}$, $\mathrm{m}), 1.04-1.10(6 \mathrm{H}, \mathrm{m}), 1.18-1.20(18 \mathrm{H}, \mathrm{d}, \mathrm{J}=7.5 \mathrm{~Hz}), 1.35-1.88(32 \mathrm{H}, \mathrm{m}), 4.18-4.29(6 \mathrm{H}, \mathrm{m})$, $7.63(1 \mathrm{H}, \mathrm{s}), 7.76(1 \mathrm{H}, \mathrm{s}) .{ }^{13} \mathrm{CNMR}\left(\mathrm{CDCl}_{3}\right): \delta 11.13,11.46,11.97,14.19,14.27,14.28,18.73$, $23.10,23.27,23.27,23.95,24.03,24.06,29.06,29.41,29.43,30.48,30.62,30.70,38.90,40.78$, $68.10,75.86,75.99,99.41,118.20,118.29,120.58,120.64,129.12,129.97,130.14,131.82$, 131.98, 134.12, 134.33, 136.79, 136.86, 137.88, 143.57, 144.89, 148.77, 151.05, 161.07, 161.10. ${ }^{19} \mathrm{FNMR}\left(\mathrm{CDCl}_{3}\right): \delta-108.72$. MALDI: calcd, 992.3; found, 992.6.

(M2b) ${ }^{1} \mathrm{HNMR}\left(\mathrm{CDCl}_{3}\right): \delta$ 0.94-1.06 $(18 \mathrm{H}, \mathrm{m}), 1.04-1.16(12 \mathrm{H}, \mathrm{m}), 1.20-1.22(36 \mathrm{H}, \mathrm{d}$, $\mathrm{J}=7.5 \mathrm{~Hz}), 1.40-1.54(26 \mathrm{H}, \mathrm{m}), 1.64-1.84(13 \mathrm{H}, \mathrm{m}), 1.86-1.92(4 \mathrm{H}, \mathrm{m}), 4.25-4.38(10 \mathrm{H}, \mathrm{m}), 7.660$ $(1 \mathrm{H}, \mathrm{s}), 7.665(1 \mathrm{H}, \mathrm{s}), 7.67(1 \mathrm{H}, \mathrm{s}), 7.94(1 \mathrm{H}, \mathrm{s}) .{ }^{13} \mathrm{CNMR}\left(\mathrm{CDCl}_{3}\right): \delta 11.18,11.49,11.50,11.59$, $12.00,14.24,14.31,14.33,14.37,18.77,23.12$, 23.31, 23.32, 23.35, 24.01, 24.08, 24.11, 24.16, $29.11,29.44,29.47,29.48,30.53,30.68,30.74,30.75,30.77,38.98,40.84,68.18,75.93,76.06$, $76.17,117.55,117.82,117.91,120.96,11.02,124.64,128.57,128.71,129.24,129.66,129.70$, $131.10,131.27,131.96,132.12,132.42,132.49,132.58,133.46,134.12,134.15,134.30,134.40$, $137.74,138.04,143.66,144.51,144.96,149.34,151.41,161.44,161.46 .{ }^{19} \mathrm{FNMR}\left(\mathrm{CDCl}_{3}\right): \delta$ 110.37. MALDI, calcd, 1516.5; found, 1516.8 .

(M2a) 1a $(0.60 \mathrm{~g}, 0.98 \mathrm{mmol})$ and $\operatorname{BrFTTBr}(0.25 \mathrm{~g}, 0.53 \mathrm{mmol})$ were measured into a round bottom flask, together with $\mathrm{Pd}\left(\mathrm{PPh}_{3}\right)_{4}(25 \mathrm{mg})$ and $\mathrm{LiCl}(0.5 \mathrm{mg})$. After three cycles of vacuum and refill with $\mathrm{Ar}$, toluene/DMF $(20 \mathrm{~mL} / 5 \mathrm{~mL})$ was added. The solution was then heated to 100 ${ }^{\circ} \mathrm{C}$ o.n. After cooling down to room temperature, the solvent was removed and the reaction mixture was purified by column chromatography of silica gel with hexane/dichloromethane $=3 / 1$ to give the final product M2a as red oil $(0.42 \mathrm{~g}, 0.35 \mathrm{mmol}$, yield $66 \%) . \delta 0.88-1.02(18 \mathrm{H}, \mathrm{m})$, 1.05-1.10 (12H, m), 1.35-1.88 (30H, m), 4.18-4.32 $(10 \mathrm{H}, \mathrm{m}), 7.36-7.38(2 \mathrm{H}, \mathrm{m}), 7.43-7.46(2 \mathrm{H}$, $\mathrm{m}), 7.56(1 \mathrm{H}, \mathrm{s}), 7.85(1 \mathrm{H}, \mathrm{s}) .{ }^{13} \mathrm{CNMR}\left(\mathrm{CDCl}_{3}\right): \delta 11.16,11.48,11.49,11.58,14.24,14.26$, $14.33,14.35,14.38,22.80,23.12,23.27,23.30,23.31,23.99,24.02,24.10,92.10,29.38,29.38$, $29.44,30.50,30.58,30.62,30.68,31.74,38.96,40.82,40.84,68.18,76.18,76.21,76.46,117.33$, $117.84,117.93,120.52,120.74,120.81,124.49,126.56,126.81,128.85,129.52,130.67,130.83$, $131.05,131.22,131.93,132.08,132.33,132.43,132.50,132.57,132.64,133.43,144.22,144.42$, 144.98, 145.44, 149.24, 151.51, 161.38. ${ }^{19} \mathrm{FNMR}\left(\mathrm{CDCl}_{3}\right): \delta$-109.34. MALDI: calcd, 1203.8; found, 1204.5 .

(M2c) M2a $(0.20 \mathrm{~g}, 0.17 \mathrm{mmol})$ was measured into a $100 \mathrm{~mL}$ round bottom flask and protected with Ar. $50 \mathrm{~mL}$ anhydrous THF was added and cooled down to $-78{ }^{\circ} \mathrm{C}$. LDA (1M, 
$0.41 \mathrm{~mL})$ was added dropwise and kept at this temperature for 1 hour. Then $\mathrm{Me}_{3} \mathrm{SnCl}(1 \mathrm{M}$, $0.5 \mathrm{~mL}$ ) was added dropwise and the reaction mixture was kept at $-78{ }^{\circ} \mathrm{C}$ for another 3 hour. Then $20 \mathrm{~mL}$ water was added to quench the reaction. After warm to r.t., the reaction mixture was poured into water and extracted with $\mathrm{Et}_{2} \mathrm{O}$. The organic layer was washed with water for three times and dried over anhydrous $\mathrm{Na}_{2} \mathrm{SO}_{4}$. Filtration and removal of the solvent gave the crude product M2c as deep red oil $(0.26 \mathrm{~g})$, which was used without further purification. ${ }^{1} \mathrm{HNMR}$ $\left(\mathrm{CDCl}_{3}\right): \delta$ 0.40-0.55 $(18 \mathrm{H}, \mathrm{t}), 0.97-1.10(30 \mathrm{H}, \mathrm{m}), 1.30-1.90(45 \mathrm{H}, \mathrm{m}), 4.20-4.36(10 \mathrm{H}, \mathrm{m})$, 7.49-7.56 (2H, m), $7.62(1 \mathrm{H}, \mathrm{s}), 7.89(1 \mathrm{H}, \mathrm{s})$.

(M3) M1 $(0.10 \mathrm{~g}, 0.10 \mathrm{mmol})$ and 2,6-bis(trimethyltin)-4,8-bis(2-ethylhexyloxy)benzo[1,2b:4,5-b']dithiophene (BDT-ditin) $(0.035 \mathrm{~g}, 0.046 \mathrm{mmol})$ were measured into a round bottom flask, together with $\mathrm{Pd}\left(\mathrm{PPh}_{3}\right)_{4}(8.2 \mathrm{mg})$. After three cycles of vacuum and refill with Ar, toluene/DMF $(8 \mathrm{~mL} / 2 \mathrm{~mL})$ was added. The solution was then heated to $100^{\circ} \mathrm{C}$ o.n. After cooling down to room temperature, the solvent was removed and the reaction mixture was purified by column chromatography of silica gel with hexane/dichloromethane $=2 / 1$ to give the final product $\mathbf{M 3}$ as deep red oil $(0.085 \mathrm{~g}, 0.037 \mathrm{~mol}$, yield $81 \%)$. ${ }^{1} \mathrm{HNMR}\left(\mathrm{CDCl}_{3}\right): \delta 0.97-1.15(48 \mathrm{H}, \mathrm{m}), 1.18-1.20$ $(36 \mathrm{H}, \mathrm{d}, \mathrm{J}=7.5 \mathrm{~Hz}), 1.35-1.98(78 \mathrm{H}, \mathrm{m}), 4.21-4.35(16 \mathrm{H}, \mathrm{m}), 7.56(2 \mathrm{H}, \mathrm{s}), 7.64(2 \mathrm{H}, \mathrm{s}), 7.91$ $(2 \mathrm{H}, \mathrm{s}) .{ }^{13} \mathrm{CNMR}\left(\mathrm{CDCl}_{3}\right): \delta 11.15,11.48,11.62,14.24,14.33,14.44,18.78,23.12,23.31,23.36$, 23.97, 24.05, 24.08, 24.11, 29.08, 29.43, 29.47, 29.86, 30.48, 30.65, 30.69, 30.73, 38.94, 40.83, $40.86,68.24,76.07,76.18,76.33,117.28,117.87,117.96,121.08,121.15,124.22,128.70$, 129.22, 129.63, 129.98, 131.09, 131.26, 132.09, 132.49, 132.96, 133.99, 134.15, 134.44, 138.12, 143.64, 144.52, 144.99, 149.29, 151.56, 161.38. ${ }^{19} \mathrm{FNMR}\left(\mathrm{CDCl}_{3}\right): \delta-112.17$. MALDI: calcd, 2271.1; found, 2271.3.

(M4) Follow the same procedure but with M1 $(0.23 \mathrm{~g}, 0.23 \mathrm{mmol})$ and M2b $(0.17 \mathrm{~g}$, $0.11 \mathrm{mmol})$, M4 was obtained as purple oil $(0.15 \mathrm{~g}, 0.049 \mathrm{mmol}$, yield $43 \%) .{ }^{1} \mathrm{HNMR}\left(\mathrm{CDCl}_{3}\right): \delta$ 0.97-1.15 (66H, m), 1.18-1.19 (36H, d, J = 7.5Hz), 1.35-1.98 (105H, m), 4.19-4.40 (22H, m), $7.51(3 \mathrm{H}, \mathrm{s}), 7.62(2 \mathrm{H}, \mathrm{s}), 7.85(1 \mathrm{H}, \mathrm{s}), 7.88(2 \mathrm{H}, \mathrm{s}) .{ }^{13} \mathrm{CNMR}\left(\mathrm{CDCl}_{3}\right): \delta 11.07,11.46,11.49$, $11.67,12.01,14.24,14.27,14.35,14.46,14.51,18.79,23.16,23.31,23.33,23.36,23.40,23.87$, 24.02 , 24.07, 24.11, 29.02, 29.43, 29.48, 29.52, 30.38, 30.61, 30.67, 30.73, 38.85, 38.94, 40.79, $40.84,40.86,68.27,75.98,76.14,76.43,117.08,117.22,117.96,118.05,117.96,121.03,124.25$, $128.70,129.17,129.30,129.48,129.86,130.09$, 131.10, 132.11, 132.56, 132.64, 132.98, 133.69, 133.88, 134.13, 134.41, 138.00, 143.61, 144.11, 144.33, 144.85, 144.99, 149.13, 151.40, 161.27.

${ }^{19}$ FNMR $\left(\mathrm{CDCl}_{3}\right): \delta-112.07,-112.02$. MALDI: calcd, 3027.3; found [M+1] ${ }^{+}, 3028.5$.

(1) Lu, L.; Zheng, T.; Xu, T.; Zhao, D.; Yu, L. Mechanistic Studies of Effect of Dispersity on the Photovoltaic Performance of PTB7 Polymer Solar Cells. Chemistry of Materials 2015, 27, 537 543.

(2) Zhong, H.; Li, C. Z.; Carpenter, J.; Ade, H.; Jen, A. K. Influence of Regio- and Chemoselectivity on the Properties of Fluoro-Substituted Thienothiophene and Benzodithiophene Copolymers. Journal of the American Chemical Society 2015, 137, 7616-7619.

(3) Liang, Y.; Feng, D.; Wu, Y.; Tsai, S.-T.; Li, G.; Ray, C.; Yu, L. Highly Efficient Solar Cell Polymers Developed via Fine-Tuning of Structural and Electronic Properties. Journal of the American Chemical Society 2009, 131, 7792-7799.

\section{HNMR Data}




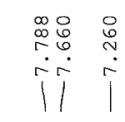
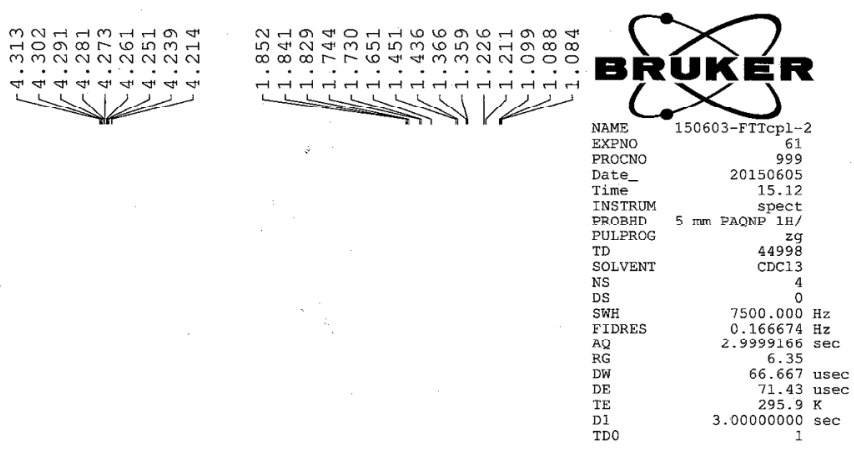

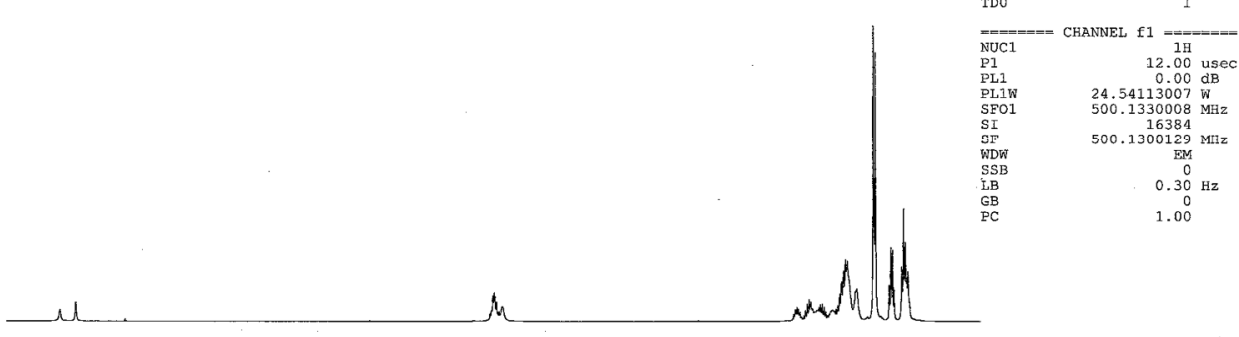
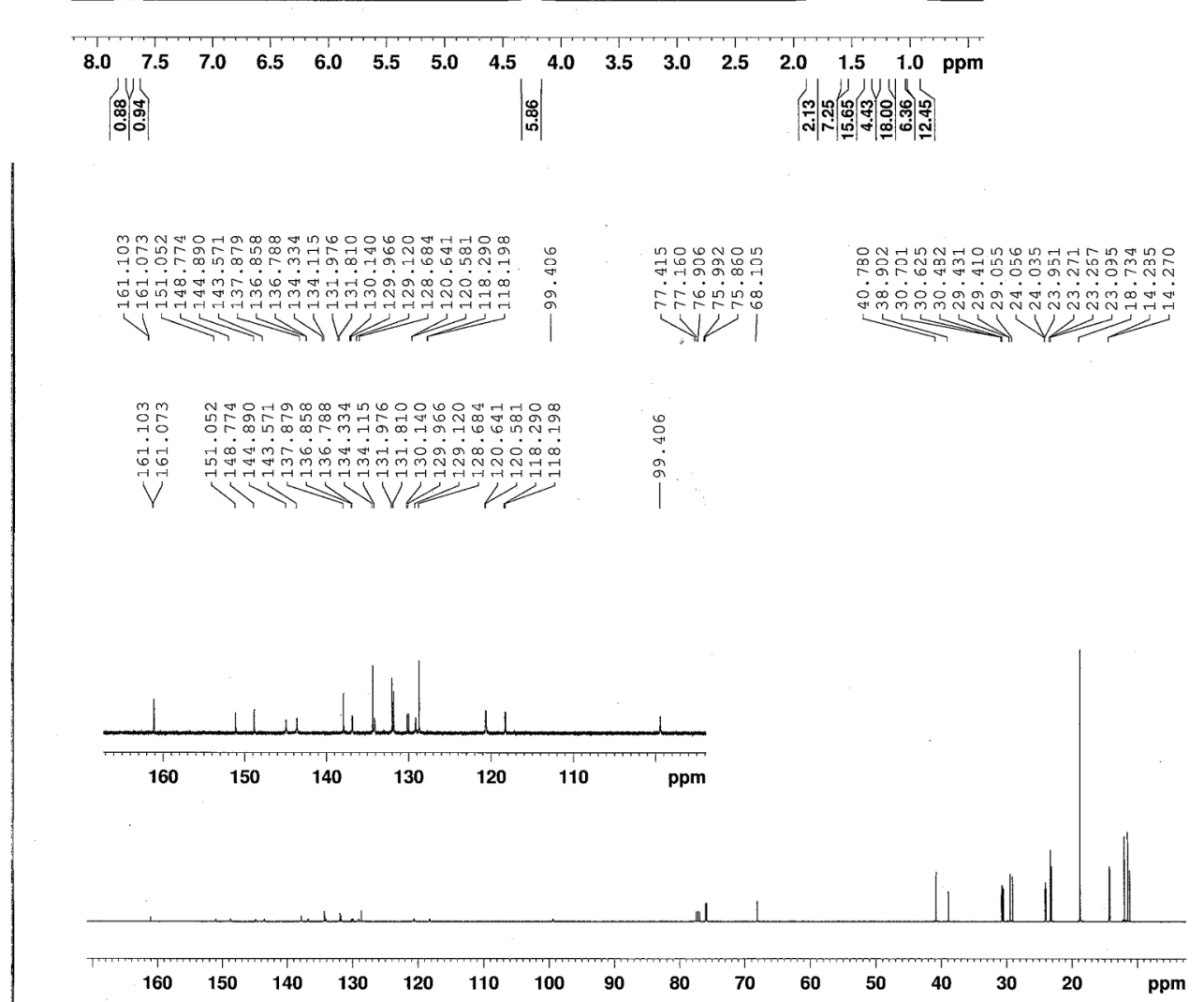

Figure S10 1H and 13C NMR spectra of M1. 


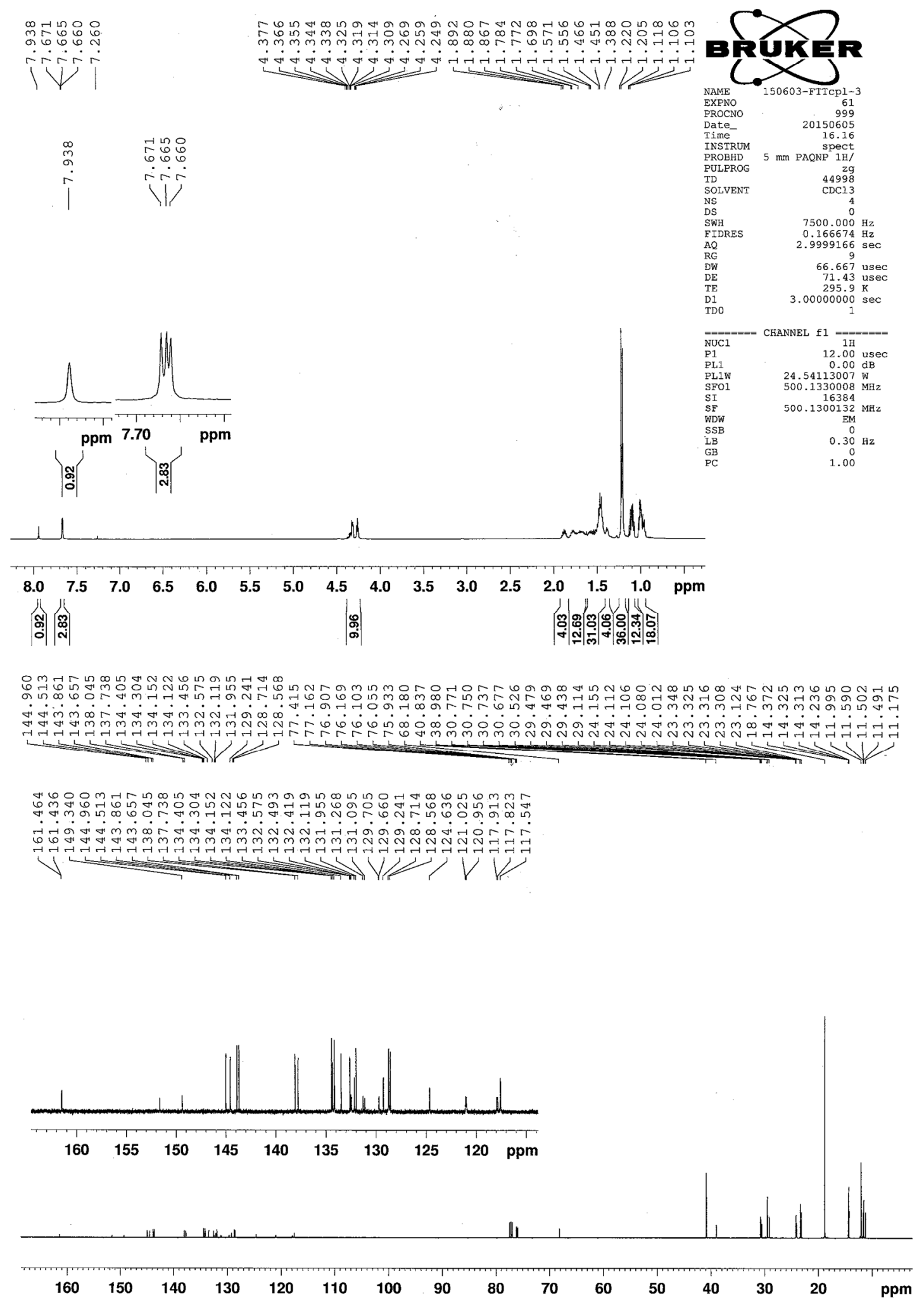

Figure S11 1H and 13C NMR spectra of M2b. 


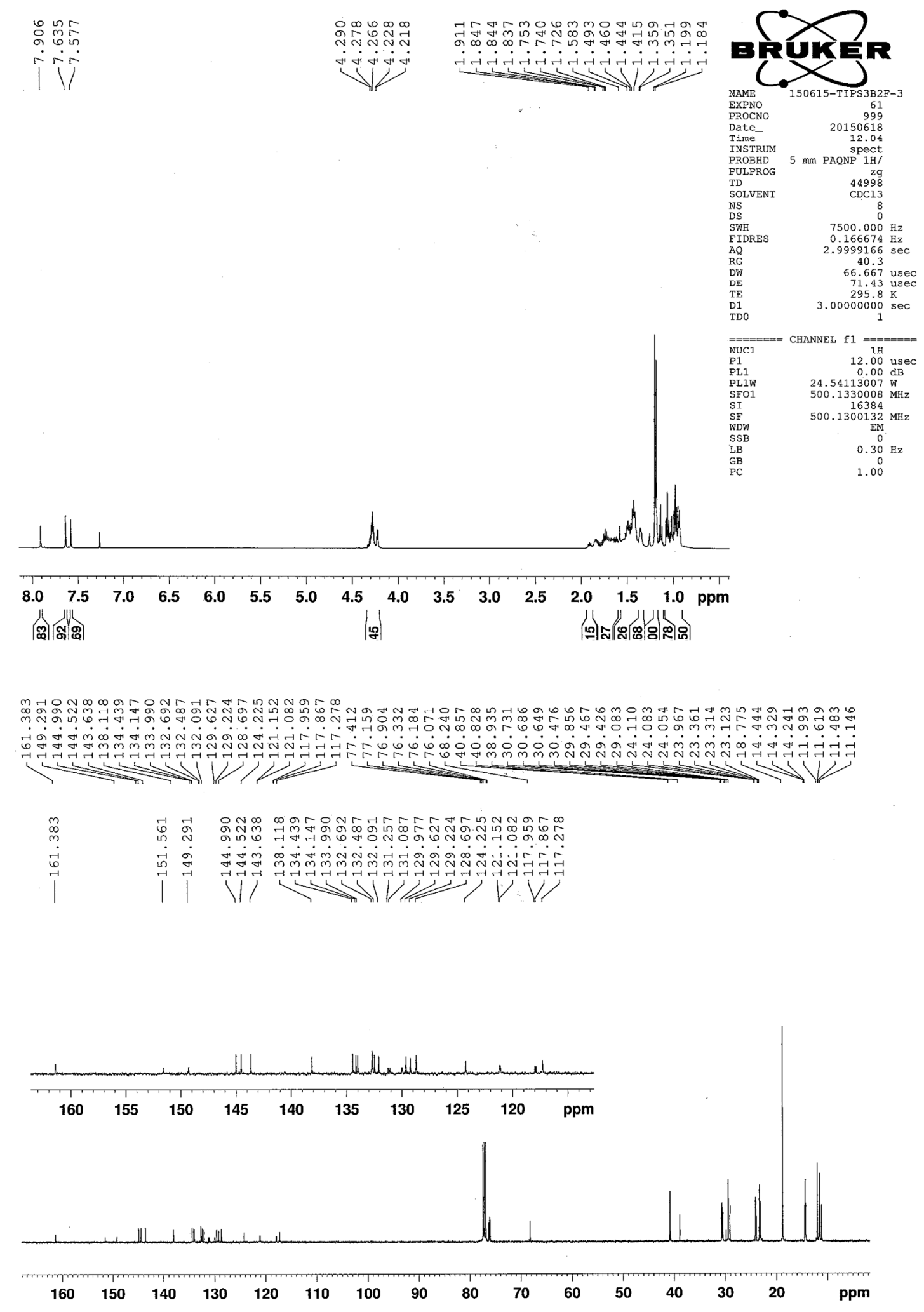

Figure S12 1H and 13C NMR spectra of M3. 

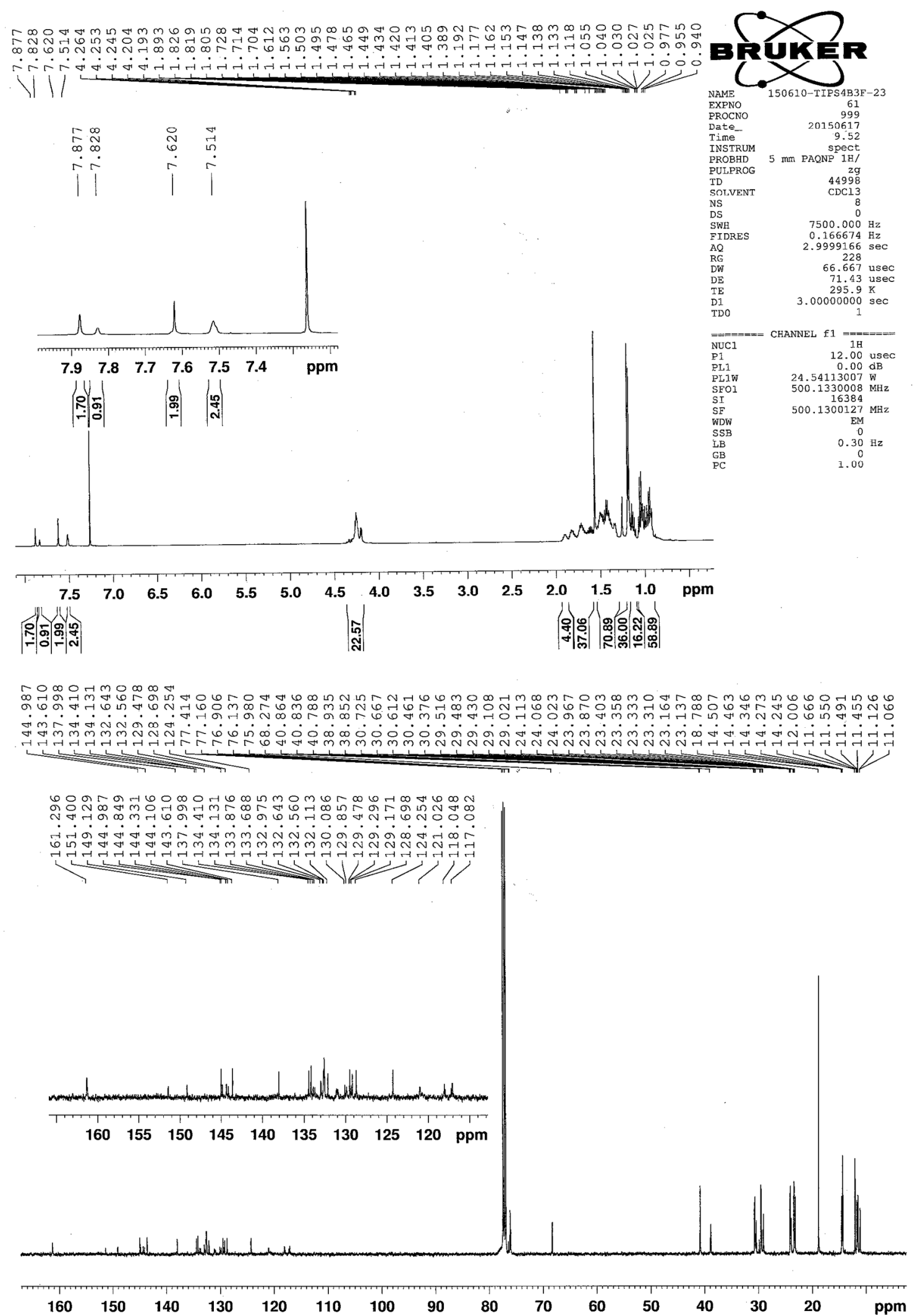

Figure S13 1H and 13C NMR spectra of M4. 

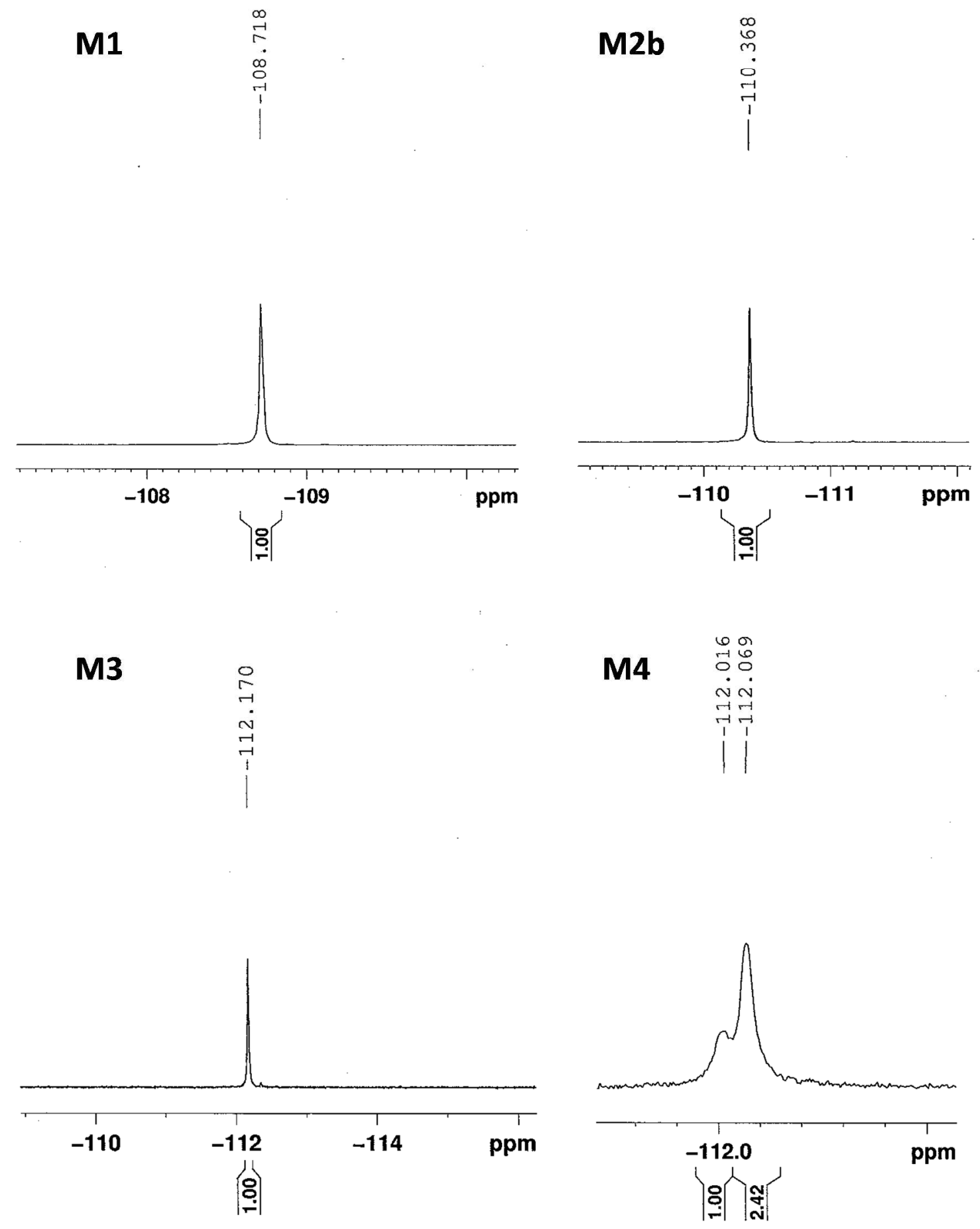

Figure S14 19F NMR spectra of M1, M2b, M3 and M4. 
(1) Vorov, O. K.; Livesay, D. R.; Jacobs, D. J. Conformational Entropy of an Ideal CrossLinking Polymer Chain. Entropy 2008, 10, 285-308.

(2) De Gennes, P. G. Introduction to Polymer Dynamics; Cambridge University Press: New York, 1990.

(3) Lu, L.; Zheng, T.; Xu, T.; Zhao, D.; Yu, L. Mechanistic Studies of Effect of Dispersity on the Photovoltaic Performance of PTB7 Polymer Solar Cells. Chem. Mater. 2015, 27, $537-$ 543. 